\title{
A Constrained Genetic Approach for Computing Material Property of Elastic Objects
}

\author{
Yong Zhang, Lawrence O. Hall, Fellow, IEEE, Dmitry B. Goldgof, Member, IEEE, and \\ Sudeep Sarkar, Senior Member, IEEE
}

\begin{abstract}
This paper presents a constrained genetic approach for reconstructing the material properties of elastic objects. The considered reconstruction problem is ill-posed and must be constrained properly so that a unique and stable numerical solution can be obtained. Qualitative prior information is incorporated using a rank-based scheme to constrain the admissible solutions. Experiments show that the proposed approach is robust when presented with noisy data and can reconstruct the elastic property accurately and reliably. In a comparison study with the deterministic Gauss-Newton methods, the constrained genetic approach also shows very consistent performance.
\end{abstract}

Index Terms-Constrained genetic algorithm (CGA), elastic property, finite-element model, inverse problem.

\section{INTRODUCTION}

\section{A. Background and Proposed Approach}

$\mathbf{R}$ ECONSTRUCTING elastic properties noninvasively is of interest to researchers in many fields. For example, identifying elasticity abnormalities in soft tissue has high potential in early cancer detection [16], [50], [29], [54], [13]. Knowing elastic properties of deformable objects is also critical for physics-based studies such as motion tracking, realistic animation, visualization, and surgical planning [35], [63], [38], [53], [14] because the quality of elastic properties could have strong impact on the performance of physical models. Recovering elastic properties has also found applications in structural damage identification, geophysical exploration, wafer engineering, robotics design, and composite material characterization [7], [3], [17], [32], [21].

The commonly used methods for elastic property reconstruction consist of three steps: 1) capture the object's deformation with various imaging modalities that use seismic wave, ultrasound, magnetic resonance, or optical cameras; 2) measure the deformation on images using the correspondence algorithms such as statistical matching or optical flow; 3) reconstruct the elastic properties from the measured deformation data. In this paper, we concentrate on the third step, assuming that the deformation data has been obtained from images.

Manuscript received September 14, 2004; revised January 25, 2005; July 30, 2005. This work was supported in part by the National Science Foundation under Grant CNS-0130768.

Y. Zhang is with the Department of Computer Science and Information Systems, Youngstown State University, Youngstown, OH 44555 USA (e-mail: yzhang@ysu.edu).

L. O. Hall, D. B. Goldgof, and S. Sarkar are with the Department of Computer Science and Engineering, University of South Florida, Tampa, FL 33620 USA (e-mail: hall@cse.usf.edu; goldgof@cse.usf.edu; sarkar@cse.usf.edu).

Digital Object Identifier 10.1109/TEVC.2005.860767
Inferring elastic properties from the observed deformation is an ill-posed inverse problem. The standard least squares approach cannot guarantee a stable solution because of the discontinuous dependence of the solution on the data. A regularization scheme such as the Tikhonov functional is therefore needed to pose a smoothness constraint. The Tikhonov functional of a nonlinear ill-posed inverse problem is very difficult to solve because its convexity can only be guaranteed very locally. In the deterministic domain, the use of gradient-based methods is common [10]. The drawback of the gradient-based methods is that they are very sensitive to the starting point and thus often stuck in local extrema. This is very true in elasticity reconstruction where the nonlinearity of inverse formulation leads to an extremely complex solution space that is characterized by numerous local plateaus.

Recently, solving inverse problems with stochastic algorithms (such as with genetic algorithms) has received much attention. The stochastic nature of genetic algorithms offers us a better chance to find the optimal solution by escaping local minima. Wong and Guan [68] used evolutionary programming to solve an adaptively regularized image restoration problem. Olmi et al. [49] reported that a genetic algorithm outperformed the Newton-Raphson method in electrical impedance tomography. Chiwiacowsky et al. [7] proposed a hybrid method that utilizes the strengths of both the Newton's method and the genetic algorithm.

Another important issue in solving an ill-posed elasticity problem is how to incorporate qualitative prior information. In addition to the quadratic function itself that poses a smoothness constraint, quite often, a special prior term is used to pull the solution in the specified direction. Unfortunately, such prior knowledge is often expressed in a qualitative form, for which most constraint handling techniques in genetic algorithms are not appropriate [5], [41]. A mechanism is therefore needed to incorporate the qualitative information into the solution strategy.

We propose using a constrained genetic algorithm (CGA) to solve the ill-posed inverse problem of reconstructing the Young's modulus of elastic objects as a distributed parameter. The proposed approach has the following features.

1) The CGA is much less sensitive to the initialization, and the strict requirement of a good starting point can be relaxed in practice. Our experiments with both the Gauss-Newton method and the CGA show that the latter is more consistent in terms of the convergence performance. 
2) Prior knowledge is incorporated through a rank table, which enables us to handle the qualitative information that cannot be readily expressed as a continuous function and its derivatives.

\section{B. Related Work}

In Table I, we summarize the related work on elastic property reconstruction. The list is by no means exhaustive. We only include the papers that are most relevant to our interest, i.e., those that deal with the computational aspect of solving an inverse elastic problem with either a deterministic or a stochastic algorithm. For studies on the direct measurement and the development of special imaging modalities such as magnetic resonance elastography, ultrasonic elastography, and optical coherence tomography, we refer to [19], [9], [13], [51], and [34].

It is apparent that most of the studies chose deterministic algorithms that use either direct or iterative inversions. Kallel and Bertrand [33] used the Newton-Raphson method combined with a finite-element model to estimate the Young's modulus of synthetic tissues. Doyley et al. [8] studied a modified iterative Newton-Raphson method. Van Houten et al. [65] proposed a multiresolution method (subzone) to improve both the efficiency and robustness. Other deterministic algorithms such as the level set method [2], the adjoint state method [46], the steepest descent search [64], and the iterative regularization [11] have also been reported. In those studies, discussions were focused on the uniqueness, accuracy, and stability of inverse solutions, while the initialization issue was rarely addressed. This could potentially be problematic because a good initial guess is very difficult to obtain in practice, especially for large-scale finite-element models.

On the other hand, in studies that used genetic algorithms [6], [7], [20], [31], [32], little detail was given about the algorithms' performance in terms of their computational efficiency. Due to the lack of quantitative comparison data for the two approaches (deterministic versus stochastic), it is often a difficult task to design an optimal solution strategy that is both robust and efficient. To this end, we will conduct several comparative experiments to examine both the robustness and the convergence rate of the genetic algorithm and the Newton-Raphson method.

Both linear and nonlinear constitutive models have been studied. The linear model is computationally attractive and usually a good approximation for many objects. We will use a linear model to demonstrate the proposed CGA. For objects that exhibit strong nonlinearity in deformation, a nonlinear model can be adopted with minor changes in the CGA.

Table I also shows that the use of a numerical model is common, even though effort has been made to experiment with actual materials. With a synthetic model, it is easy to control the complexity of inverse problems by changing the model's configuration, so that the efficacy of the recovery algorithms can be thoroughly investigated. We will also use numerical models.

\section{PROBLEM Formulation}

Our approach to reconstruct the Young's modulus of elastic objects is based on the output least squares formula [10]. We give a brief review of the forward problem using both the partial differential equations and the discretized finite-element model. We then use the forward model to formulate the inverse problem as a regularized functional.

\section{A. Forward Problem of Linear Elasticity}

By the principle of conservation of momentum [18], the deformation of a solid body caused by external forces can be stated as a boundary value problem represented by the following partial differential equation:

$$
\begin{array}{rlrl}
\nabla \cdot \boldsymbol{\sigma}+\rho \mathbf{f}_{\mathbf{b}} & =0, & \text { in } \Omega=\Gamma_{1}+\Gamma_{2} \\
\mathbf{u} & =\overline{\mathbf{u}}, & & \text { on } \Gamma_{1} \\
\frac{\partial \mathbf{u}}{\partial \mathbf{n}} & =\overline{\mathbf{g}}, & & \text { on } \Gamma_{2}
\end{array}
$$

where $\sigma=\left(\sigma_{x x}, \sigma_{y y}, \sigma_{z z}, \tau_{x y}, \tau_{y z}, \tau_{z x}\right)$ is the stress tensor, $\mathbf{u}=(u, v, w)$ is the displacement vector, $\rho$ is the mass density, $\mathbf{f}_{\mathbf{b}}$ is the body force, $\overline{\mathbf{u}}$ and $\overline{\mathrm{g}}$ are the prescribed displacement and force (Dirichlet and Neumann conditions) on the boundary $\left(\Gamma_{1}, \Gamma_{2}\right)$ that define the modeling domain $\Omega$, and $\mathbf{n}$ is the outward unit normal on the boundary.

For a linear elastic object, the stress tensor $\sigma$ is related to the strain tensor and material properties through the constitutive equation

$$
\begin{gathered}
\boldsymbol{\sigma}=\mathbf{C e} \\
{\left[\begin{array}{l}
\sigma_{x x} \\
\sigma_{y y} \\
\sigma_{z z} \\
\tau_{x y} \\
\tau_{y z} \\
\tau_{z x}
\end{array}\right]=\left[\begin{array}{lllllll}
c_{11} & c_{12} & c_{13} & c_{14} & c_{15} & c_{16} \\
c_{21} & c_{22} & c_{23} & c_{24} & c_{25} & c_{26} \\
c_{31} & c_{32} & c_{33} & c_{34} & c_{35} & c_{36} \\
c_{41} & c_{42} & c_{43} & c_{44} & c_{45} & c_{46} \\
c_{51} & c_{52} & c_{53} & c_{54} & c_{55} & c_{56} \\
c_{61} & c_{62} & c_{63} & c_{64} & c_{65} & c_{66}
\end{array}\right]\left[\begin{array}{c}
e_{x x} \\
e_{y y} \\
e_{z z} \\
\gamma_{x y} \\
\gamma_{y z} \\
\gamma_{z x}
\end{array}\right]}
\end{gathered}
$$

where $\mathbf{e}=\left(e_{x x}, e_{y y}, e_{z z}, \gamma_{x y}, \gamma_{y z}, \gamma_{z x}\right)$ is the strain tensor and $\mathbf{C}=\left[c_{i j}\right]$ is the elastic coefficient tensor and is assumed to be constant for small deformations. For isotropic materials with Young's modulus $E$ and Poisson's ratio $\nu$, the constitutive equation is further simplified to

$$
\begin{gathered}
{\left[\begin{array}{l}
\sigma_{x x} \\
\sigma_{y y} \\
\sigma_{z z} \\
\tau_{x y} \\
\tau_{y z} \\
\tau_{z x}
\end{array}\right]=\frac{E}{(1+\nu)(1-2 \nu)}} \\
\cdot\left[\begin{array}{cccccc}
1-\nu & \nu & \nu & 0 & 0 & 0 \\
\nu & 1-\nu & \nu & 0 & 0 & 0 \\
\nu & \nu & 1-\nu & 0 & 0 & 0 \\
0 & 0 & 0 & \frac{1}{2}-\nu & 0 & 0 \\
0 & 0 & 0 & 0 & \frac{1}{2}-\nu & 0 \\
0 & 0 & 0 & 0 & 0 & \frac{1}{2}-\nu
\end{array}\right]\left[\begin{array}{c}
e_{x x} \\
e_{y y} \\
e_{z z} \\
\gamma_{x y} \\
\gamma_{y z} \\
\gamma_{z x}
\end{array}\right] .
\end{gathered}
$$

For an object of small deformation, its strain tensor can be approximated from the displacement vector by

$$
\mathbf{e}=\left[\begin{array}{ccc}
\frac{\partial u}{\partial x} & \frac{1}{2}\left(\frac{\partial u}{\partial y}+\frac{\partial v}{\partial x}\right) & \frac{1}{2}\left(\frac{\partial u}{\partial z}+\frac{\partial w}{\partial x}\right) \\
\frac{1}{2}\left(\frac{\partial v}{\partial x}+\frac{\partial u}{\partial y}\right) & \frac{\partial v}{\partial y} & \frac{1}{2}\left(\frac{\partial v}{\partial z}+\frac{\partial w}{\partial y}\right) \\
\frac{1}{2}\left(\frac{\partial w}{\partial x}+\frac{\partial u}{\partial z}\right) & \frac{1}{2}\left(\frac{\partial w}{\partial y}+\frac{\partial v}{\partial z}\right) & \frac{\partial w}{\partial z}
\end{array}\right]
$$


TABLE I

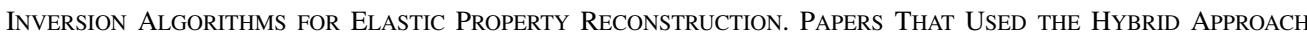
Combining the Deterministic and Genetic Algorithms are Classified as Genetic. Papers That CONSIDERED GEOMETRICAL OR MATERIAL LiNEARITIES TO SimPlify THE FORWARD MODEL ARE Classified as linear. No More Than Two Papers Were Selected From the Same RESEARCH GROUP (Authors) UNLESS THE ALGORITHMS USED WERE SigNIFICANTLy DifFERENT

\begin{tabular}{|c|c|c|c|c|}
\hline Authors & Date & Algorithms & Models & Experiments (Material) \\
\hline Aglyamov et al [1] & 2004 & Deterministic & Linear & Thrombosis \\
\hline Ameur et al $[2]$ & 2004 & Deterministic & Linear & Numerical \\
\hline Arns et al $[3]$ & 2002 & Deterministic & Linear & Porous media \\
\hline Barbone et al [4] & 2004 & Deterministic & Linear & Numerical \\
\hline Chiroiu et al $[6]$ & 2000 & Genetic & Nonlinear & Crystal lattice \\
\hline Chiwiacowsky et al [7] & 2004 & Genetic & Linear & Numerical \\
\hline Doyley et al $[8]$ & 2000 & Deterministic & Linear & Numerical \\
\hline Engl et al [11] & 2003 & Deterministic & Linear & Polymer \\
\hline Eskin et al [12] & 2002 & Deterministic & Linear & Numerical \\
\hline Franca et al $[17]$ & 2004 & Deterministic & Linear & Silicon wafer \\
\hline Furukawa et al [20] & 1997 & Genetic & Nonlinear & Cr-Mo steel \\
\hline Garboczi et al [21] & 1995 & Deterministic & Linear & Numerical \\
\hline Hori et al $[27]$ & 2003 & Deterministic & Linear & Numerical \\
\hline Huang et al [28] & 2001 & Deterministic & Nonlinear & Numerical \\
\hline Ji et al $[30]$ & 2004 & Deterministic & Linear & Numerical \\
\hline John et al [31] & 2003 & Genetic & Linear & Pelvis bone \\
\hline Joukhadar et al [32] & 1997 & Genetic & Linear & Numerical \\
\hline Kallel et al $[33]$ & 1996 & Deterministic & Linear & Numerical \\
\hline Kirkpatrick et al [34] & 2002 & Deterministic & Linear & Tissues \\
\hline Maurice et al [36] & 2004 & Deterministic & Linear & Vessel walls \\
\hline Miga [39] & 2003 & Deterministic & Linear & Numerical \\
\hline Muthupillai et al [43] & 1995 & Deterministic & Linear & Gel \\
\hline Nakamura et al [44] & 1993 & Deterministic & Linear & Numerical \\
\hline Oberai et al [46] & 2003 & Deterministic & Linear & Numerical \\
\hline O'Donnell et al [47] & 2004 & Deterministic & Nonlinear & Heart tissue \\
\hline Oliphant et al [48] & 2001 & Deterministic & Linear & Phantom \\
\hline Pellot-Barakat et al [55] & 2004 & Deterministic & Linear & Phantom \\
\hline Plewes et al $[56]$ & 2000 & Deterministic & Linear & Numerical \\
\hline Raghavan et al [57] & 1994 & Deterministic & Linear & Numerical \\
\hline Samani et al $[60]$ & 2001 & Deterministic & Linear & Breast tissues \\
\hline Sumi et al $[62]$ & 1998 & Deterministic & Linear & Numerical \\
\hline Tsap et al [64] & 1998 & Deterministic & Nonlinear & Skin \\
\hline Van Houten et al [65] & 1999 & Deterministic & Linear & Numerical \\
\hline Zhu et al $[70]$ & 2003 & Deterministic & Linear & Numerical \\
\hline
\end{tabular}

Substituting (4) and (5) into (1), the final governing equation of elastic deformation used in the Young's modulus reconstruction becomes

$$
\nabla \cdot\left[\lambda(\nabla \cdot \mathbf{u}) \mathbf{I}+G \nabla \mathbf{u}+G(\nabla \mathbf{u})^{T}\right]+\rho \mathbf{f}_{\mathbf{b}}=0
$$

where $G$ and $\lambda$ are the Lamé material property constants, which can be computed from Young's modulus $E$ and Poisson's ratio $\nu$ by

$$
\begin{aligned}
G & =\frac{E}{2(1+\nu)} \\
\lambda & =\frac{\nu E}{(1+\nu)(1-2 \nu)} .
\end{aligned}
$$

To numerically solve (6), the finite-element method is used to discretize the partial differential equation into a linear matrix equation [71]. The finite-element formulation of partial differential equations can be derived using a variational method. Assuming an elastic body in static equilibrium without inertia and dynamic vibrations, the principle of virtual work states that the external work equals the internal work

$\sum_{i=1}^{m} \int_{V} \overline{\mathbf{e}}_{i}^{T} \boldsymbol{\sigma}_{i} d V=\sum_{i=1}^{m} \int_{V} \overline{\mathbf{u}}_{i}^{T} \mathbf{f}_{\mathbf{b} i} d V+\sum_{i=1}^{m} \int_{S} \overline{\mathbf{u}}_{i}^{T} \mathbf{f}_{\mathbf{s} i} d S+\sum_{j=1}^{k} \overline{\mathbf{u}}_{j}^{T} \mathbf{f}_{\mathbf{p}_{j}}$

where $\mathbf{f}_{\mathbf{b} i}$ is the body force within an element, $\mathbf{f}_{\mathbf{s} i}$ is the surface traction, and $\mathbf{f}_{\mathbf{p}_{j}}$ is the point load. $\overline{\mathbf{u}}_{i}$ denotes the virtual displacement, $\overline{\mathbf{e}}_{i}$ denotes the virtual strain, $m$ is the number of elements, and $k$ is the number of point loads.

To satisfy the requirement of strain compatibility, an interpolation function $\mathbf{H}$ (shape function) is chosen that is continuous within the element

$$
\mathbf{d}_{i}=\mathbf{H}_{i} \mathbf{u}
$$

where $\mathbf{d}_{i}=\left[d_{x}, d_{y}, d_{z}\right]^{T}$ is the displacement within each element.

The strain-displacement and stress-strain relationships in the discrete matrix forms are

$$
\begin{aligned}
\mathbf{e}_{i} & =\mathbf{B}_{i} \mathbf{u} \\
\boldsymbol{\sigma}_{i} & =\mathbf{C}_{i} \mathbf{e}_{i}
\end{aligned}
$$


where $\mathbf{B}$ is obtained by differentiating and combining the rows of $\mathbf{H}$.

By combining (9)-(12) and canceling the transpose of nodal displacement $\mathbf{u}^{T}$, we obtain the formulation for assembling system matrices of a finite-element model

$$
\begin{aligned}
& {\left[\sum_{i=1}^{m} \int_{V} \mathbf{B}_{i}^{T} \mathbf{C}_{i} \mathbf{B}_{i} d V\right] \mathbf{u}} \\
& \quad=\sum_{i=1}^{m} \int_{V} \mathbf{H}_{i}^{T} \mathbf{f}_{\mathbf{b} i} d V+\sum_{i=1}^{m} \int_{S} \mathbf{H}_{i}^{T} \mathbf{f}_{\mathbf{s} i} d S+\sum_{j=1}^{k} \mathbf{f}_{\mathbf{p}_{j}}
\end{aligned}
$$

or in a more concise form

$$
\mathbf{K u}=\mathbf{F}
$$

where $\mathbf{K}$ is the stiffness matrix and $\mathbf{F}$ is the generalized force

$$
\begin{aligned}
\mathbf{K} & =\sum_{i=1}^{m} \int_{V} \mathbf{B}_{i}^{T} \mathbf{C}_{i} \mathbf{B}_{i} d V \\
\mathbf{F} & =\sum_{i=1}^{m} \int_{V} \mathbf{H}_{i}^{T} \mathbf{f}_{\mathbf{b} i} d V+\sum_{i=1}^{m} \int_{S} \mathbf{H}_{i}^{T} \mathbf{f}_{\mathbf{s} i} d S+\sum_{j=1}^{k} \mathbf{f}_{\mathbf{p}_{j}} .
\end{aligned}
$$

Since material properties such as Young's modulus $E$ and Poisson's ratio $\nu$ are embedded in the stiffness matrix $\mathbf{K}$ through the material matrix $\mathbf{C}$, we rewrite the linear (14) with respect to $(E, \nu)$ explicitly

$$
\mathbf{K}(E, \nu) \mathbf{u}=\mathbf{F} .
$$

Because we are interested in reconstructing Young's modulus only, by assuming that Poisson's ratio can be approximated as a constant $(0.45)$ for compressible materials, the final discrete forward equation to be used for the Young's modulus reconstruction is

$$
\mathbf{K}(E) \mathbf{u}=\mathbf{F} .
$$

\section{B. Inverse Problem of Reconstructing the Young's Modulus}

To cast Young's modulus reconstruction as an optimization problem, we utilize the forward equation directly in an ouput least squares formulation. We rewrite the forward (16) to derive a nonlinear inverse operator equation

$$
Q(E)=\mathbf{K}(E)^{-1} \mathbf{F}=\mathbf{b}
$$

where $\mathbf{b}$ denotes the measurement data that includes both the displacement and the boundary force and $Q$ is an operator that represents the forward model and links the measurement data (b) with the parameter to be recovered $(E)$. We assume that all components of the displacement vector $\mathbf{u}=(u, v, w)$ are known throughout the modeling domain (all nodal points of finite-element mesh). We also assume that the boundary forces (Neumann condition) are known to ensure the uniqueness of the inverse solution. As a result, the data vector can be expressed as $\mathbf{b}=\left[\overline{\mathbf{g}}_{1}, \overline{\mathbf{g}}_{2}, \ldots, \overline{\mathbf{g}}_{k}, \overline{\mathbf{u}}_{1}, \overline{\mathbf{u}}_{2}, \ldots, \overline{\mathbf{u}}_{n}\right]$, where $(\overline{\mathbf{g}}, \overline{\mathbf{u}})$ denote the known boundary force and the displacement vector, $k$ is the number of nodes upon which boundary forces are exerted, and $n$ is the total number of nodes. Note that although the forward model (16) is a linear equation, its corresponding inverse operator (17) is highly nonlinear and the data vector $\mathbf{b}$ is often corrupted by noise, which makes the inverse problem very difficult to solve.

Reconstructing the Young's modulus by solving (17) is an ill-posed inverse problem. Because of the discontinuous dependence of solution $E$ on noisy data $\mathbf{b}$, prior knowledge is needed to constrain the solution

$$
E=\arg \cdot \min \left\{L_{1}(Q(E), \mathbf{b})+\alpha L_{2}\left(E, E_{\text {prior }}\right)\right\}
$$

where $L_{1}$ measures the compatibility of the outputs from the forward model $Q(E)$ to the noisy data $\mathbf{b}, L_{2}$ measures the distance between the computed solution $E$ and the expected solution $E_{\text {prior }}$ defining a priori knowledge on $E$, and $\alpha$ is a weighting coefficient that balances the influences of measurement data and prior knowledge on the final solution.

Using the Euclidean norm, we reformulate (18) as

$$
\begin{aligned}
E & =\arg \cdot \min \{U(E)\} \\
& =\arg \cdot \min \left\{\|Q(E)-\mathbf{b}\|^{2}+\alpha\left\|W\left(E-E_{\text {prior }}\right)\right\|^{2}\right\}
\end{aligned}
$$

It is clear that $U(E)$ is the Tikhonov functional in its variational form with $\alpha$ as the regularization parameter. $W$ is the smoothness matrix that represents the discretized gradient, Laplacian, or higher order derivatives.

The Tikhonov functional can be minimized with either the deterministic gradient-based local methods such as the Newton-Raphson method and its variants, or the stochastic global methods such as the simulated annealing and genetic algorithms. The main difficulty of minimizing the Tikhonov functional of a nonlinear operator equation lies in the fact that the solution space does not posses the property of global convexity. As a result, without a good initialization, the gradient-based methods will likely diverge or converge prematurely on a local minimum.

For comparison with the genetic algorithms, we briefly describe the Gauss-Newton method (GNM) for solving the Tikhonov functional. We assume that the nonlinear operator $Q: E \rightarrow \mathbf{b}$ is continuously Fréchet differentiable in the Hilbert space. We then linearize the Tikhonov functional $U(E)$ locally around a point $E_{k}$ in the solution space

$\psi(E)=\left\|Q\left(E_{k}\right)+D\left(E_{k}\right)\left(E-E_{k}\right)-\mathbf{b}\right\|^{2}+\alpha\left\|W\left(E-E_{\text {prior }}\right)\right\|^{2}$

where $\psi$ denotes the linearized Tikhonov functional around the point $E_{k}$ and $D(E)$ is the Fréchet derivative of the nonlinear operator.

The linearized Tikhonov functional can be minimized by the first-order necessary condition

$$
\begin{array}{r}
\nabla \psi(E)=0 \\
D^{T}\left(E_{k}\right)\left(Q\left(E_{k}\right)+D\left(E_{k}\right)\left(E-E_{k}\right)-\mathbf{b}\right) \\
+\alpha W^{T} W\left(E-E_{\text {prior }}\right)=0 .
\end{array}
$$




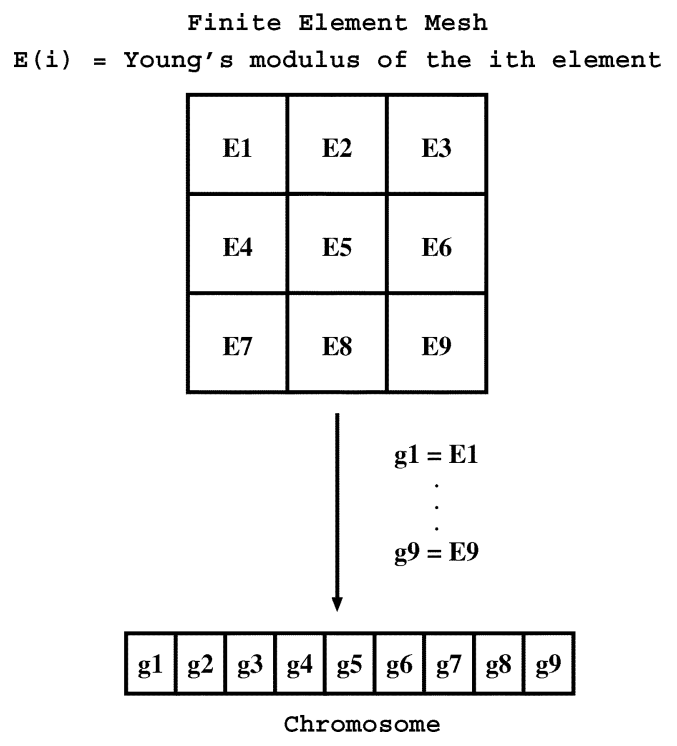

Fig. 1. Genetic coding of the Young's modulus in a finite-element model.

An iterative formula for estimating a new solution $E_{k+1}$ from the solution of previous step can be obtained by rearranging the above equation

$$
\begin{aligned}
E_{k+1}= & E_{k}+\triangle E \\
\triangle E= & {\left[D^{T}\left(E_{k}\right) D\left(E_{k}\right)+\alpha W^{T} W\right]^{-1} } \\
& \cdot\left[D^{T}\left(E_{k}\right)\left(\mathbf{b}-Q\left(E_{k}\right)\right)-\alpha W^{T} W\left(E_{k}-E_{\text {prior }}\right)\right] .
\end{aligned}
$$

More detailed information on solving the nonlinear ill-posed problems and the related numerical implementation issues in the finite dimensional space can be found in [45] and [10].

\section{Constrained Genetic Algorithm}

To solve the inverse problem of reconstructing the Young's modulus with a constrained genetic algorithm, we address the following issues: 1) encoding a two-/three-dimensional (2-D/3-D) spatial variable such as $E(x)$ in a one-dimensional (1-D) genetic computational unit; 2) expressing and incorporating qualitative prior knowledge in the genetic algorithm as a rank-based constraint; 3) balancing contributions from the measurement data which have uncertainties and the prior knowledge through stochastic ranking.

\section{A. Genetic Encoding}

Given the finite-element model of a deformed object, its Young's modulus can be interpreted as a chromosome in a CGA. The Young's modulus value of each element is encoded as a gene in the chromosome through a one-to-one mapping function (Fig. 1). As a result, if the finite-element model has $N$ elements, the corresponding chromosome would have $N$ genes. Each chromosome in the population pool represents a possible Young' modulus distribution. If dynamic meshing (multigrid) is used in the finite-element model, more sophisticated encoding schemes have to be considered that allow the size and shape of chromosomes to change adaptively during the evolution [23], [24], [61].
This one-to-one mapping function is straightforward to implement and works well for a 1-D finite-element model. However, information about the spatial connections among the neighboring elements in a 2-D/3-D finite-element mesh is completely lost in this encoding scheme. Computations that rely on spatial information cannot be performed properly. For example, in many studies, our interest is to identify only a few isolated areas of abnormal Young's modulus values from the background of relatively uniform Young's modulus distribution, and the information about genes' spatial distribution is needed in a CGA to accomplish the task efficiently. More importantly, the smoothing effect represented by the derivative operators in the deterministic regularization cannot be realized in a rank-based CGA without such spatial information. To overcome those shortcomings of one-to-one mapping, a mechanism is designed to remember the original spatial connections among neighboring elements. An auxiliary link table is created for all chromosomes. Table II shows an example that uses a four-neighbor connection for the 2-D quadrilateral mesh in Fig. 1. Similarly, a three-neighbor connection can be considered for a triangle mesh. This link table will be used in the constrained mutation operation. Because the Young's modulus has continuous values that can vary in the range of several orders of magnitude, a real-valued (double) encoding approach is used [15].

\section{B. Rank-Based Constraint}

In the stochastic framework of genetic computation, the illposedness of a nonlinear inverse problem implies many local plateaus in the landscape of the admissible solution space. By having a diverse population pool, genetic algorithms can explore a much wider solution space than the gradient methods and thus have a better chance to find the optimal solution by escaping the local minima. The ill-posed nature of the inverse problem also shows up as highly unstable solutions that are physically meaningless. To overcome those numerical difficulties associated with the ill-posedness, various constraints that represent prior knowledge must be imposed. In a CGA, constraints can take the form of penalty functions [5], which are equivalent to the regularization stabilizers or the preconditioners in the deterministic methods [10]. It should be pointed out that other competitive approaches of solving constrained optimization problems have been proposed, where the constraints and the objective function are handled separately through a multimembered mechanism [66], [37].

We formulate the objective function to be minimized by a CGA as a combination of the fitness function and the penalty function

$$
O B J(E)=\|Q(E)-\mathbf{b}\|_{n}^{2}+\theta P(E)
$$

where $Q(E)$ denotes the output of forward model, $\mathbf{b}$ is the data vector, $n$ is the number of nodes on which the measurement is made, $P(E)$ is the penalty function, and $\theta$ is a weight coefficient.

In studies of spline and surface fitting, a smoothness constraint imposed on the spline and the surface is often formulated as a quadratic integral functional such as the Sobolev norm. Similarly, Young's modulus as a distributed parameter can be 
Prior Rank Table

\begin{tabular}{|c|c|c|c|c|}
\hline \multicolumn{2}{|c|}{ Prior Knowledge } & Element II & $\mathrm{E}(\mathrm{kPa})$ & \multirow{2}{*}{$\begin{array}{c}\text { Prior Rank } \\
1 \\
\end{array}$} \\
\hline \multirow{2}{*}{$\begin{array}{l}\text { (1) } \\
\text { low }\end{array}$} & \multirow{2}{*}{\begin{tabular}{|l|} 
(2) \\
high
\end{tabular}} & (2) & high & \\
\hline & & \begin{tabular}{|l|}
$(3)$ \\
\end{tabular} & mid & 2 \\
\hline \multirow{2}{*}{$\begin{array}{l}\text { (3) } \\
\text { mid }\end{array}$} & (4) & (1) & low & 3,4 \\
\hline & low & (4) & low & 3,4 \\
\hline
\end{tabular}

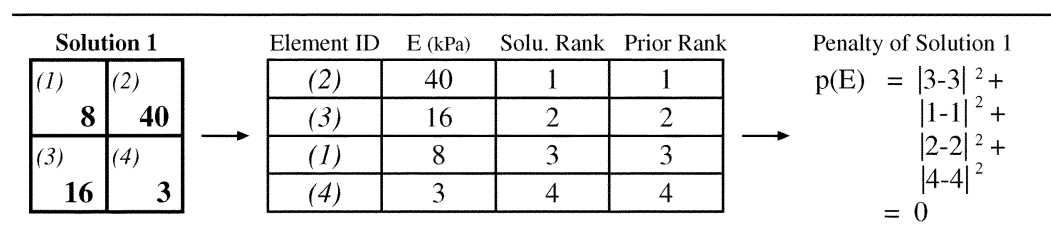

\begin{tabular}{|c|c|c|c|c|c|}
\hline \multicolumn{2}{|c|}{ Solution 2} & Element ID & $\mathrm{E}(\mathrm{kPa})$ & Solu. Rank & Prior Rank \\
\hline \multirow{2}{*}{ (1) $\begin{array}{ll} & \\
& 4\end{array}$} & \multirow{2}{*}{\begin{tabular}{|l|} 
(2) \\
22
\end{tabular}} & (4) & 35 & 1 & 3 \\
\hline & & (2) & 22 & 2 & 1 \\
\hline \multirow[t]{2}{*}{ (3) } & \multirow{2}{*}{$\begin{array}{r}\text { (4) } \\
35\end{array}$} & (3) & 6 & 3 & 2 \\
\hline & & (1) & 4 & 4 & 4 \\
\hline
\end{tabular}

Penalty of Solution 2 $\mathrm{p}(\mathrm{E})=|4-4|^{2}+$ $|2-1|^{2}+$ $|3-2|^{2}+$ $|1-3|^{2}$

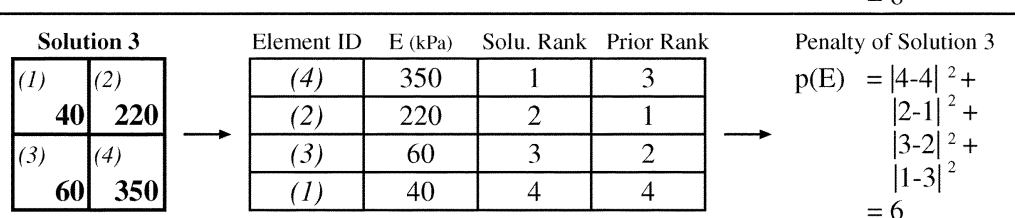

Fig. 2. The rank-based scheme for incorporating qualitative prior information. The numbers in the parentheses denote element ID and the bold numbers at the lower right corner of elements represent the Young's modulus $(\mathrm{kPa})$.

regarded as a spatial function that possesses certain degrees of continuity and the smoothness constraints can be realized as

$$
P(E)=\int_{R}|\nabla E(x)|^{2} d x
$$

where $\nabla$ could be any derivative operator such as the gradient or the Laplacian.

Knowledge about the relative Young's modulus distribution can be obtained from either an expert's visual assessment or from low-level image cues such as intensity, color, and texture. This type of prior knowledge is often expressed qualitatively and is not suited for penalty functions that are composed of continuous differential operators. To utilize the qualitative prior knowledge in a genetic algorithm, we propose an alternative rank-based method to compute the penalty function. In each possible solution (chromosome), elements (genes) will be ranked based on their relative Young's modulus values and their positions will be recorded in a sorted rank table. Similarly, we transform the qualitative prior knowledge into another rank table. For each element of the model, we compute the difference of its ranked positions in the two rank tables. We then sum up the rank discrepancy of all elements to represent the distance between the solution and the prior knowledge

$$
P(E)=\sum_{i=1}^{m}\left\|r_{i}-R_{i}\right\|^{2}
$$

where $r_{i}$ is the rank position of element $i$ in the rank table for the solution, $R_{i}$ is the rank position of element $i$ in the rank table based on prior knowledge, and $m$ is the number of elements.

Fig. 2 illustrates the ranking scheme with a simple 2-D model of four elements. As the qualitative prior knowledge, the Young's modulus value of each element is labeled as "high," "mid," and "low." This qualitative information is then transformed into a rank table where elements are sorted in descending order from "high" to "low." If $n$ elements $(n>1)$ have the same label, they all can have $n$ potential rank positions, which will be determined by their counterparts in the solution rank table. For instance, both element (1) and element (4) are labeled as "low," therefore their rank position can be either three or four. In Solution 1, the ranks of elements (2) and (3) in the solution table match exactly with their ranks in the table of prior knowledge. However, for element (1), its rank in the solution table is three, while its rank in prior knowledge table is three or four. In the case of multiple ranks, we select the value that is closest to its counterpart in the solution table, which is three for element (1). Similarly, for element (4), we select four from its multiple prior rank of three or four. The final penalty value for Solution 1 is zero $(P(E)=0)$. In contrast, Solution 2 has a distribution that is quite different from the specified prior knowledge and thus a shuffled rank table, which leads to a higher penalty value of six.

One potential problem with this rank-based penalty function is that two solutions of the same rank table are penalized equally, even if their absolute Young's modulus values are quite different. As demonstrated in Fig. 2, Solution 3 has a Young's modulus distribution that is ten times higher than that of Solution 2. But their solution rank tables are exactly the same and therefore receive the same amount of penalty of six. This problem is related to the nonuniqueness of the inverse solution and can be resolved by incorporating both displacement and force in the data vector $\mathbf{b}$. In other words, Solutions 2 and 3 will have different fitness functions because of their different $\mathbf{b}$ and $Q(E)$ values. This ambiguity can also be partially resolved by introducing another penalty function that specifies a range (both the upper bound and the lower bound) within which the Young's modulus of an element is allowed to vary. 
This rank-based approach has the advantage that it is intrinsically piecewise and helps preserve the parameter discontinuity (although a strong smoothness constraint can still be imposed in the areas of little Young's modulus variation). This approach is particularly suitable for studies that aim at identifying and quantifying property abnormalities. In our previous studies on burn scar assessment [64], [69], qualitative prior knowledge was collected from physicians who isolate and rate the scars based on a relative rating scale. Automatic methods for extracting the information directly from image intensity, texture, and color can also be considered.

\section{Balancing the Fitness and Penalty Scores by Stochastic Ranking}

In the constrained objective function (26), the fitness and penalty terms are computed on different quantities. The fitness is measured as difference of displacement (meter), while the penalty is based on the difference of rank orders (unitless). An optimal weight coefficient $\theta$ is needed to balance their contributions. If $\theta$ is too small, the data noise will not be penalized enough and the resulting solution becomes unstable. If $\theta$ is too large, solution will be forced into a smoothed prior space and most of the data signals, i.e., the information used to infer the Young's modulus, will be lost due to overpenalization. In the deterministic domain, several choices are available for determining the optimal regularization parameter. If the noise level of observation data is known, methods based on the discrepancy principle [42] such as the Miller method [40] can be considered. In case that information about the data noise is not available, heuristic methods such as generalized cross-validation [67] or the L-curve [25] method are commonly used. However, those methods are deterministic and not suited for handling rank-based qualitative data.

In a recent study on the constrained evolutionary optimization, Runarsson and Yao [58] presented a stochastic method to strike a balance between the fitness and penalty functions, without the need of computing the weight coefficient explicitly. Determination of an optimal $\theta$ is related to the dynamic and adaptive ranking of individual chromosome in a population. Ranking is based on the relative dominance of either the fitness function or the penalty function between two adjacent individuals. The balance of dominance is achieved by introducing a bubble-sort-like dynamic ranking procedure for an individual to win a comparison. We found this stochastic method is well suited for handling qualitative prior knowledge in elasticity reconstruction. We refer readers to [58] for detailed discussions on the method and related implementation issues.

\section{Genetic Operators}

To minimize the objective function (26), we need to specify several important genetic operators. It should be emphasized that, in the next section that investigates the performance of different algorithms [genetic algorithm (GA), CGA, and GNM], we used the same parameter settings for the GA and the CGA (mutation, crossover, selection and replacement, elitism, as well as the encoding scheme). The main difference is that the GA does not have a constraint handling mechanism, and hence does not incorporate the qualitative prior information in its search.
TABLE II

LINK TABLE FOR MAINTAINING ORIGINAL SPATIAL CONNECTIONS

\begin{tabular}{|c|c|l|}
\hline Gene ID & Values & 4-Neighbor Connections \\
\hline \hline $\mathrm{g} 1$ & $\mathrm{E} 1$ & $\mathrm{~g} 2=\mathrm{E} 2, \mathrm{~g} 4=\mathrm{E} 4$ \\
\hline $\mathrm{g} 2$ & $\mathrm{E} 2$ & $\mathrm{~g} 1=\mathrm{E} 1, \mathrm{~g} 3=\mathrm{E} 3, \mathrm{~g} 5=\mathrm{E} 5$ \\
\hline $\mathrm{g} 3$ & $\mathrm{E} 3$ & $\mathrm{~g} 2=\mathrm{E} 2, \mathrm{~g} 6=\mathrm{E} 6$ \\
\hline $\mathrm{g} 4$ & $\mathrm{E} 4$ & $\mathrm{~g} 1=\mathrm{E} 1, \mathrm{~g} 5=\mathrm{E} 5, \mathrm{~g} 7=\mathrm{E} 7$ \\
\hline $\mathrm{g} 5$ & $\mathrm{E} 5$ & $\mathrm{~g} 2=\mathrm{E} 2, \mathrm{~g} 4=\mathrm{E} 4, \mathrm{~g} 6=\mathrm{E} 6, \mathrm{~g} 8=\mathrm{E} 8$ \\
\hline $\mathrm{g} 6$ & $\mathrm{E} 6$ & $\mathrm{~g} 3=\mathrm{E} 3, \mathrm{~g} 5=\mathrm{E} 5, \mathrm{~g} 9=\mathrm{E} 9$ \\
\hline $\mathrm{g} 7$ & $\mathrm{E} 7$ & $\mathrm{~g} 4=\mathrm{E} 4, \mathrm{~g} 8=\mathrm{E} 8$ \\
\hline $\mathrm{g} 8$ & $\mathrm{E} 8$ & $\mathrm{~g} 5=\mathrm{E} 5, \mathrm{~g} 7=\mathrm{E} 7, \mathrm{~g} 9=\mathrm{E} 9$ \\
\hline $\mathrm{g} 9$ & $\mathrm{E} 9$ & $\mathrm{~g} 6=\mathrm{E} 6, \mathrm{~g} 8=\mathrm{E} 8$ \\
\hline
\end{tabular}

1) Mutation: We used a standard Gaussian mutation operator

$$
g_{j}^{*}=g_{j}+\sigma \mathcal{N}(0,1) g_{j}
$$

where $g_{j}$ is the value of gene $j$ before the mutation, $g_{j}^{*}$ is the value of gene $j$ after the mutation, $\mathcal{N}(0,1)$ is a random Gaussian number $($ mean $=0$, standard deviation $=1$ ), and $\sigma$ is the mutation step size. $\sigma$ is initialized to be 0.2 in the experiments. The dynamic control of mutation step size is determined by a predefined decay rate $\tau$ as

$$
\sigma(k+1)=\tau \sigma(k)
$$

where $k$ is the generation counter. $\tau$ is usually set in the range of 0.99-1.0. A smaller value of $\tau$ may help speed up the convergence rate but has the risk of premature convergence. In our experiments, we used a $\tau$ value of 0.997 . Other than Gaussian mutation, we also considered other types of real-coded mutation operators such as uniform mutation and step mutation. Those mutation approaches did not show any advantage over Gaussian mutation in terms of both solution accuracy and computational efficiency.

We experimented with the method of dynamically setting the mutation probability $\left(P_{m}\right)$ based on population statistics and did not find significant improvement in terms of the solution accuracy. Therefore, we set $P_{m}$ to a fixed value of 0.2 in all the following experiments. One of the purposes of having a relatively high mutation rate is to maintain the population diversity and prevent premature convergence. To utilize the spatial connectivity among finite elements as recorded in the link table (Table II), each gene will be compared with its neighboring genes after the mutation. If the gene has a Young's modulus value that is much higher/lower than the maximum/minimum of its neighboring genes, the gene will be assigned a mean value of its neighbors. This process has the effect of partially restoring the smoothness property of the quantitative regularization functional that is missed in the rank-based penalty function (28).

2) Crossover: We also found that the one-point crossover operator and the multiple-point crossover operator performed equally well, at least for this particular inverse problem setting. So we used the one-point crossover operator in all of the following experiments. The crossover probability $\left(P_{c}\right)$ is fixed to 0.7 . The one-point crossover function is implemented in a traditional fashion: children are generated by joining two parents at a randomly selected crossover position and then swapping each side. 
3) Parent Selection and Replacement: The parent selection operator is implemented as tournament selection $(\mathrm{k}=2)$. We also experimented with a wide range of replacement ratios (0.05-1.0), i.e., the percentage of parent population to be replaced by new chromosomes. Given a population of size $S$ and a replacement ratio of $r$, the number of parents to be replaced is: $N=r S$. Smaller replacement ratios $(<0.3) \mathrm{did}$ not yield satisfying results because of the lack of contributions from new chromosomes to the population diversity. However, no significant difference was observed with replacement ratios ranging from 0.5 to 1.0 . On the other hand, the higher the replacement ratio, the longer the simulation time. Therefore, we chose a replacement ratio of 0.8 for the experiments.

In [58], it was shown that the stochastic ranking probability $\left(P_{f}\right)$ in the range of $(0.45-0.475)$ gave the best result for 13 benchmark functions. We tested two $P_{f}$ values $(0.45$ and 0.475$)$ in our experiments and did not observe noticeable improvement or degradation in the algorithm's performance. So we used a $P_{f}$ value of 0.45 in all of the following comparison studies.

An elitism strategy is also enabled during the replacement operation, in which some elite members of the old generation are chosen to survive to the next generation without competition (potentially being replaced by a better offspring). Given the genetic parameters specified above, trial tests with different elite ratios $(1 \%-15 \%)$ showed that $3 \%$ gave a slightly better result on average (although very marginal). So we chose an elite ratio of $3 \%$ in all the experiments.

The pseudocode for the constrained genetic algorithm is as follows:

1 /* parameter values and initialization* /

$2 S /$ * population size */

$3 r=0.8 / *$ replacement ratio */

$4 N=r S / *$ replacement size */

$5 \tau=0.997 /$ * mutation decay rate */

$6 \sigma=0.2 / *$ initial mutation step size */

$7 P_{c}=0.7 / *$ one-point crossover probability */

$8 P_{f}=0.45 / *$ stochastic ranking probability */

9 for $i=1$ to population $\mathrm{S}(0)$

10 chromosome (i) = a value from a Gaussian distribution

11 end for

$12 /$ * start the iteration ( $\mathrm{k}$ is the iteration counter) * /

13 for $\mathrm{k}=1$ to maximum generation

14 for $\mathrm{i}=1$ to population $S(\mathrm{k})$

$15 \mathrm{E}(\mathrm{i})=$ chromosome (i)

16 call forward finite-element model with E (i) compute fitness and penalty functions for chromosome

\section{end}

19 rank the whole population $S(\mathrm{k})$ with stochastic ranking $\left(P_{f}\right)$

20 pick a set of parents from population $S(\mathrm{k})$ through tournament selection use one-point crossover to generate $N$ offspring with a probability of $P_{c}$

for $\mathrm{i}=1$ to $N$ offspring

Mutate chromosome (i) : $\mathrm{g}=\mathrm{g}+\sigma \mathcal{N}(0,1) \mathrm{g}$

$\mathrm{E}(\mathrm{i})=$ chromosome (i)

E (i) chromosome (i)

end

pick $N$ parents from population $S(\mathrm{k})$

through tournament selection

for potential replacement)

with stochastic ranking $\left(P_{f}\right)$

for $\mathrm{i}=1$ to $N$

for $\mathrm{j}=1$ to $N$

if rank of parent (i) $\leq$ rank of offspring (j)

then

remove offspring $(j)$

end

end

a new population $S(\mathrm{k}+1)$

check the stopping criterion ((37))

end call forward finite-element model with

compute fitness and penalty functions for

(top 3\% elite members will not be picked

rank $N$ picked parents and $N$ offspring

replace parent (i) with offspring (j)

update mutation step size: $\sigma(\mathrm{k}+1)=\tau \sigma(\mathrm{k})$

\section{EXPERIMENTAL RESULTS}

\section{A. Experiments With GA, CGA, and GNM}

A synthetic numerical model is used to demonstrate the efficacy of the constrained genetic algorithm. The forward model is a 2-D thin shell $(5 \mathrm{~cm}$ by $5 \mathrm{~cm})$ and is discretized to 61 nodes and 100 triangle elements (Fig. 3). A small square at the upper left corner of the model is the abnormal area and has a higher Young's modulus value of $250 \mathrm{kPa}$. The rest of background elements have a uniform Young's modulus of $50 \mathrm{kPa}$. The element type is three-node triangular shell without out-of-plane deformation ( $w$ component in $z$ direction). A linear scheme is used in the interpolation (shape) functions

$$
\begin{aligned}
u(x, y) & =H_{i} u_{i}+H_{j} u_{j}+H_{k} u_{k} \\
v(x, y) & =H_{i} v_{i}+H_{j} v_{j}+H_{k} v_{k} \\
H_{i}(x, y) & =\frac{1}{2 A}\left[\left(x_{j} y_{k}-x_{k} y_{j}\right)+\left(y_{j}-y_{k}\right) x+\left(x_{k}-x_{j}\right) y\right] \\
H_{j}(x, y) & =\frac{1}{2 A}\left[\left(x_{k} y_{i}-x_{i} y_{k}\right)+\left(y_{k}-y_{i}\right) x+\left(x_{i}-x_{k}\right) y\right] \\
H_{k}(x, y) & =\frac{1}{2 A}\left[\left(x_{i} y_{j}-x_{j} y_{i}\right)+\left(y_{i}-y_{j}\right) x+\left(x_{j}-x_{i}\right) y\right]
\end{aligned}
$$

where $H$ denotes the interpolation function, $A$ is the area of element, $(i, j, k)$ are used to index three nodal positions, and $(x, y)$ represent the location in a global 2-D coordinate [Fig. 3(c)].

Boundary conditions are specified as follows. Forces (concentrated loads) are applied to the nodes of top boundary $(0.5 \mathrm{~N}$ on each node). Displacement constraints (Dirichlet type) are fixed to zero on the bottom boundary [Fig. 3(a)]. Those 


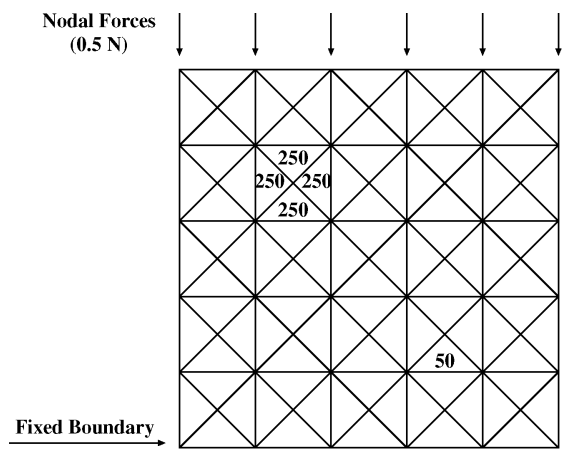

(a)

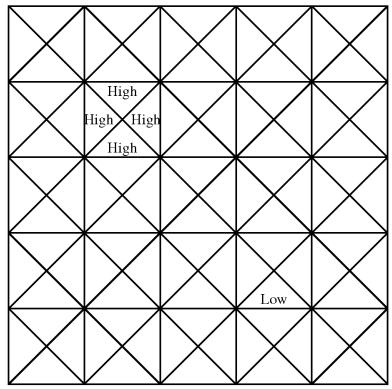

(b)

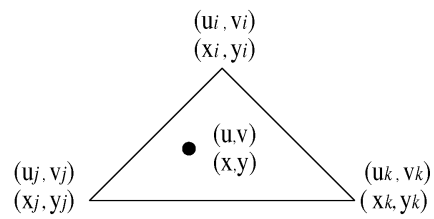

(c)

Fig. 3. The synthetic forward finite-element model of a 2-D elastic object and the qualitative prior knowledge. In (a), the Young's modulus of the background area is $50 \mathrm{kPa}$. In (b), the background area is labeled as "low." (c) illustrates the linear interpolation scheme of triangle element used in (31)-(35). (u,v) are displacement components in a 2-D coordinate, $(x, y)$ denotes the location of a point, and $(i, j, k)$ are used to index three nodes. (a) Forward model, (b) prior knowledge, and (c) triangle element

boundary conditions are used to generate noise-free displacement data on each node. The data vector $\mathbf{b}$ includes forces of top boundary nodes as well as displacements of all nodes other than those on the bottom boundary.

Three algorithms - a regular GA, CGA, and the deterministic GNMs - were tested with the noise-free data to study to what degree the Young's modulus in the abnormal area can be reproduced. The data vector includes both displacement and force to ensure the uniqueness of the inverse solution. As prior knowledge, four elements in the upper left square were identified as a potential abnormal area. These four elements were labeled as "high" and other elements in the background labeled as "low" [Fig. 3(b)].

We then added 3\% white noise to the noise-free data. Again, the GA, CGA, and GNM were studied using the noisy data to assess the performance. For this small 2-D model, we found that a population size of 50 is large enough for the GA and CGA to converge to a good solution with less than 300 generations. To provide statistical information of their performance under realistic conditions (noisy data), 30 simulations were carried out for each algorithm with noisy data.

A Gaussian distribution with a mean of $50 \mathrm{kPa}$ was used as the initial condition for all the experiments. The regularization parameter of GNM was set to 1.0E-16, and the smoothness matrix was the zero-order finite difference matrix, i.e., the identity matrix. If necessary, a higher order matrix can be used. For example, for an evenly spaced solution vector of four elements, the first-order finite difference matrix is

$$
\left[\begin{array}{rrrr}
1 & -1 & 0 & 0 \\
-1 & 2 & -1 & 0 \\
0 & -1 & 2 & -1 \\
0 & 0 & -1 & 1
\end{array}\right]
$$

Since the higher order matrices have a strong tendency to oversmooth the vector, we used the identity matrix in all the experiments.

One important issue in the design of a property recovery algorithm is the stopping criterion. For GA and CGA, we choose a rule that is based on the population similarity

$$
\left|O B J_{\text {best }}-O B J_{\text {average }}\right|<\text { tolerance }
$$

where $O B J_{\text {best }}$ denotes the best solution of the current population in terms of the objective function value and $O B J_{\text {average }}$ represents the average solution of the same population. A simulation will be stopped if the difference between the two values is below the tolerance. This stopping criterion has the advantage that it allows more consistent measure of the algorithm's performance. Based on many trial tests, we choose a tolerance value of 0.001 .

The stopping rule for GNM is defined by

$$
\frac{\|\operatorname{grad}(E)\|}{\left\|\psi\left(E_{k}\right)\right\|}<\text { tolerance }
$$

where $\psi(E k)$ is the linearized Tikhonov functional with respect to the current solution $E_{k}$ and $\operatorname{grad}(E)$ is the gradient operator of Tikhonov functional

$$
\begin{aligned}
\operatorname{grad}(E)=D^{T}\left(E_{k}\right)\left(Q\left(E_{k}\right)+\right. & \left.D\left(E_{k}\right)\left(E-E_{k}\right)-\mathbf{b}\right) \\
& +\alpha W^{T} W\left(E-E_{\text {prior }}\right)
\end{aligned}
$$

A GNM simulation is stopped if the above criterion is met or a maximum Gauss-Newton iteration number is reached (100 for this experiment). The tolerance was set to $1.0 \mathrm{e}-8$ for GNM experiments. This tolerance value is relatively stringent. A good initial solution guess usually allows the algorithm to converge with less than 20 iteration steps, while a poor initial point results in the maximum iteration number to be reached (diverge).

A solution is considered diverged if one of the two conditions is true: 1) more than half of the elements in the abnormal area have a recovery error that is larger than 0.5 and 2) more than $5 \%$ of the background elements have a recovery error that is larger than 0.5 . The recovery error is defined as $\left|E_{r}-E_{t}\right| / E_{t}$, where $E_{r}$ and $E_{t}$ are the recovered and true property values, respectively.

Examples of recovered Young's modulus are shown in Fig. 4. Given the noise-free data, all algorithms were able to find a near-optimal solution with the abnormal area well defined in terms of both the geometry and absolute values. The results from the CGA and GNM are almost identical. Given the noise corrupted data, both GNM and CGA still successfully identified the abnormal area, although the boundaries were blurred due to the smoothing effect. However, the GA failed to find an acceptable solution as evidenced by the multiple abnormal areas [Fig. 4(f)] that do not exist in the forward model. 


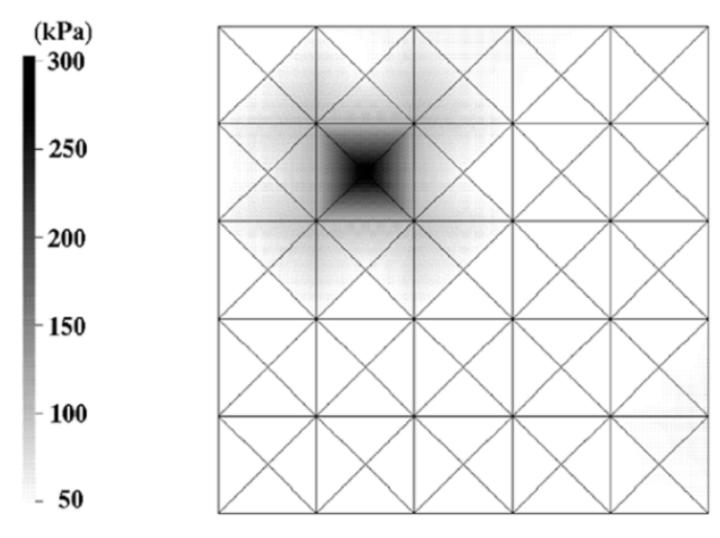

(a)

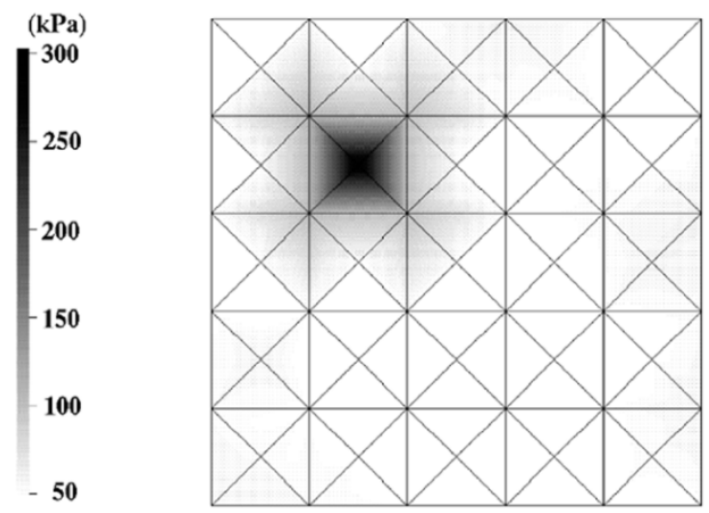

(c)

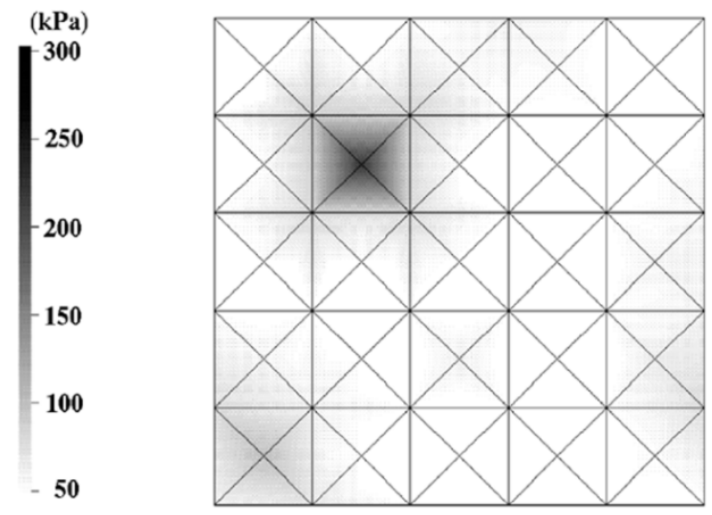

(e)

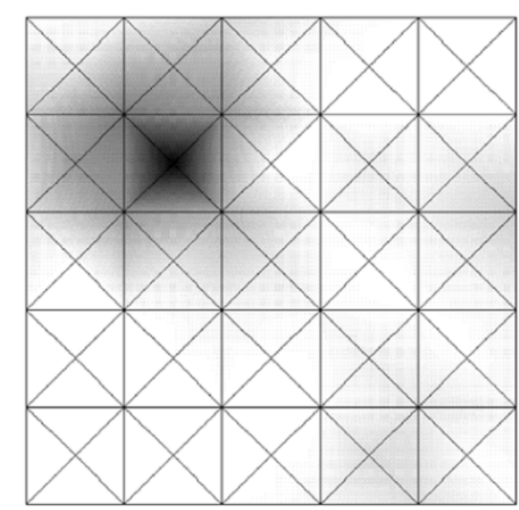

(b)

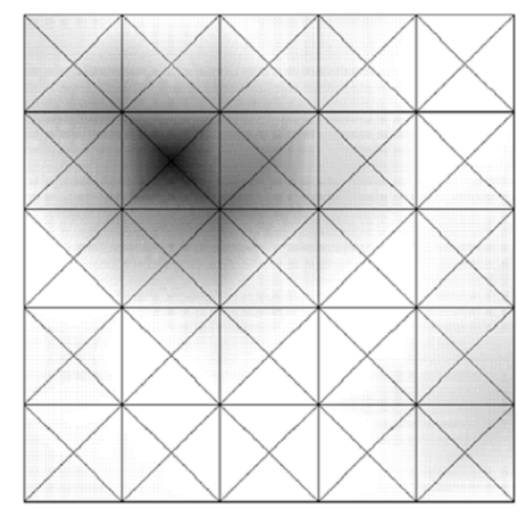

(d)

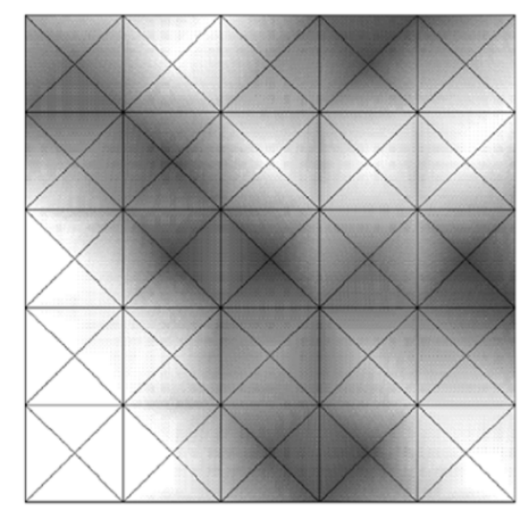

(f)

Fig. 4. Reconstructed Young's modulus by GA, CGA, and GNM. The figures are plotted using grayscale, with black and white indicating high and low Young's modulus values, respectively. The figures were generated using the General Mesh Viewer visualization software [22] that may add certain degrees of smoothing effect on the Young's modulus distribution (the Young's modulus value inside an element is constant). (a) GNM with 0\% data noise, (b) GNM with 3\% data noise, (c) CGA with $0 \%$ data noise, (d) CGA with 3\% data noise, (e) GA with $0 \%$ data noise, and (f) GA with 3\% data noise.

The statistical data from experiments with noisy data are given in Table III. All of the CGA and GNM simulations produced good solutions (100\% convergence ratio), while none of the GA simulations converged. This is also reflected in the number of evaluations of the objective function and the simulation time. CGA and GNM simulations show very stable and dense distributions (small standard deviations) and converged quickly. In contrast, the GA's performance is characterized by high standard deviations as well as the large number of evaluations and long simulation times that are almost an order of magnitude higher than that of CGA and GNM.

Table IV presents the recovered Young's modulus in terms of two representative values

$$
E_{a}=\sum_{i=1}^{I} E_{i}, \quad E_{b}=\sum_{j=1}^{J} E_{i}
$$


TABLE III

Performance of GA, CGA, ANd GNM for Noisy Data. For Each Algorithm, 30 Simulations Were Conducted. The PERformance Is Reported in Terms of the Number of EVAluations of ObJective Function, the Simulation Time, And the Convergence Ratio. The Convergence Ratio Is COMPuted as the Number OF CONVERGED CASES Divided By THE TOTAL Number OF Simulations. (SD $\rightarrow$ STANDARD DeViation)

\begin{tabular}{|c|c|c|c|c|c|c|c|c|c|c|c|c|}
\hline \multirow{2}{*}{ Algorithm } & \multicolumn{4}{|c|}{ Number of Evaluations } & \multicolumn{4}{c|}{ Simulation Time (min) } & Converg. \\
& \cline { 2 - 12 } & Min & Max & Mean & Median & SD & Min & Max & Mean & Median & SD & Ratio \\
\hline GA & 10450 & 23500 & 16255 & 15325 & 4104 & 33 & 120 & 69 & 67 & 25 & $0 \%$ \\
\hline CGA & 1429 & 3243 & 1945 & 1853 & 339 & 6 & 17 & 10 & 9 & 3 & $100 \%$ \\
\hline GNM & 1050 & 2150 & 1653 & 1600 & 275 & 4 & 11 & 7 & 7 & 1 & $100 \%$ \\
\hline
\end{tabular}

TABLE IV

Recovered Property Values With GA, CGA, and GNM for Noisy Data. Statistical Data Are CAlculated Using All 30 Simulations for EaCH Algorithm

\begin{tabular}{|c|c|c|c|c|c|c|c|c|c|c|c|c|}
\hline \multirow[t]{2}{*}{ Algorithm } & \multicolumn{6}{|c|}{ Abnormal area, $E_{a}(\mathrm{kPa})$} & \multicolumn{6}{|c|}{ Background, $E_{b}(\mathrm{kPa})$} \\
\hline & Min & $\operatorname{Max}$ & Mean & Median & $\mathrm{SD}$ & True & Min & $\operatorname{Max}$ & Mean & Median & $\mathrm{SD}$ & true \\
\hline GA & 83 & 956 & 134 & 161 & 202 & 250 & 22 & 193 & 84 & 91 & 47 & 50 \\
\hline CGA & 177 & 313 & 212 & 207 & 39 & 250 & 51 & 54 & 52 & 52 & 2 & 50 \\
\hline GNM & 186 & 248 & 219 & 220 & 22 & 250 & 44 & 68 & 56 & 55 & 6 & 50 \\
\hline
\end{tabular}

where $E_{a}$ is the averaged value in the abnormal area and $E_{b}$ is the averaged value in the background. $I$ and $J$ are the number of elements in the abnormal area and the background, respectively. It is clear that the solutions of CGA and GNM are close to the true solution in both the abnormal area and background, and more importantly, they are stable and consistent. But there is a wide range of variations in GA solutions, caused by the diverged cases.

One concern in using the rank-based CGA for property reconstruction is the quality of prior knowledge. For example, if the prior knowledge does not correspond to the true Young's modulus distribution very well, how much will the final solution be affected? We conducted an experiment with slightly biased prior knowledge. The model configuration, initial condition, and noisy data were the same as those in the previous experiments. The biased prior and the reconstructed Young's modulus are shown in Fig. 5. A rectangle of four elements was identified as the potential abnormal area and was labeled "high" incorrectly, but still close to the true abnormality square. It can be seen that the shape of the reconstructed abnormal area is influenced by the biased prior, although the CGA still managed to find the true abnormality square. The prior knowledge has a tendency to attract the inverse solution toward itself. In applications, we do not expect the prior knowledge to be severely biased, but its side-effect should be corrected as much as possible.

It is worth noting that there are two basic types of prior involved. One is the smoothness prior that is implicit in the quadratic penalty term and the other one is explicitly specified as the ranked prior table. The former is a global constraint, while the latter is local in nature. Therefore, the influence of a bad local prior cannot be remedied by adjusting the global regularization parameter or stochastic ranking in CGA. Study is needed to find more sophisticated and effective methods so that the biased prior knowledge can be handled properly.

\section{B. Experiments With CGA and GNM}

There have been concerns about the advantage and disadvantage of genetic algorithms over the traditional gradient decent methods, especially the computational efficiency for large-scale problems. To understand how various factors might affect the

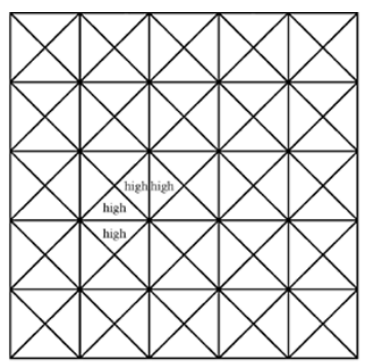

(a)

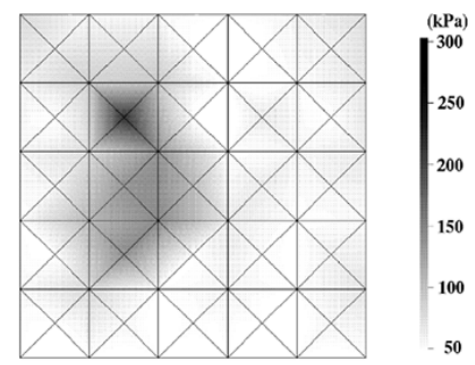

(b)
Fig. 5. Reconstructed Young's modulus by the CGA with biased prior. The same noisy data (3\% noise) was used. The prior labels four elements as "high," which are inconsistent with the true solution. The final solution is clearly influenced by the biased prior. (a) Biased prior knowledge and (b) result of CGA with $3 \%$ data noise.

performance of a CGA in solving inverse problems, we carried out another set of experiments using both the CGA and GNM.

The forward model is similar to the one that we used in the previous experiments, except a much larger parameter space of 900 elements (Fig. 6), and hence it is more difficult to find an optimal solution of the corresponding inverse problem. The model has a size of 10 by $10(\mathrm{~cm})$ and is meshed with quadrilateral elements. Twenty-four elements in the center of the model are the abnormal area with a Young's modulus value of $400 \mathrm{kPa}$, and the background elements have a lower Young's modulus value of $50 \mathrm{kPa}$. The element type used is four-nodes quadrilateral shell. Isoparametric mapping and the Lagrange formula are used in the interpolation (shape) functions

$$
\begin{aligned}
u(x, y) & =H_{i} u_{i}+H_{j} u_{j}+H_{k} u_{k}+H_{l} u_{l} \\
v(x, y) & =H_{i} v_{i}+H_{j} v_{j}+H_{k} v_{k}+H_{l} v_{l} \\
H_{i}(s, t) & =\frac{1}{4}(1-s)(1-t) \\
H_{j}(s, t) & =\frac{1}{4}(1+s)(1-t) \\
H_{k}(s, t) & =\frac{1}{4}(1+s)(1+t) \\
H_{l}(s, t) & =\frac{1}{4}(1-s)(1+t)
\end{aligned}
$$


Nodal Force $(0.4 \mathrm{~N}$

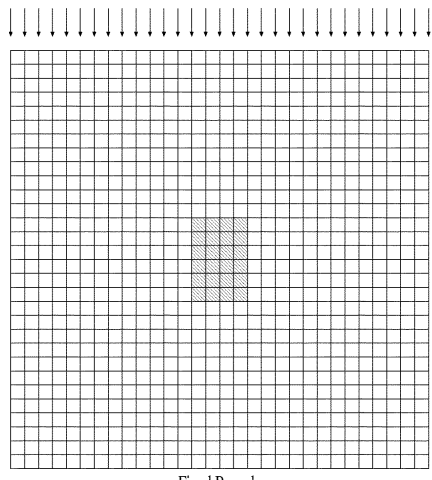

$\prod_{400 \mathrm{kPa}}^{\text {Fixed Boundary }}$

(a)

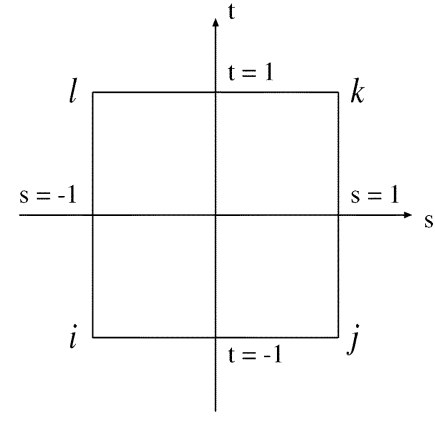

(b)
Fig. 6. The configuration of the forward model: number of elements $=900$, number of nodes $=961$, number of elements in the abnormal area $=24$. (b) Illustrates the interpolation scheme of the quadrilateral element used in (39)-(44). $(u, v)$ are displacement components, $(x, y)$ denotes the location in the global coordinate system, $(s, t)$ represents the local coordinates within an element, and $(i, j, k, l)$ are used to index four nodes. (a) Forward model and (b) quadrilateral element.

where $H$ denotes the interpolation function, $(i, j, k, l)$ are used to index four nodal positions, $(s, t)$ denotes the local coordinates of the quadrilateral element, and $(x, y)$ represents the global coordinates [see Fig. 6(b)].

The boundary conditions were specified as follows. The model was compressed by forces exerted on the top boundary ( $0.4 \mathrm{~N}$ on each node), while the bottom boundary was fixed (Dirichlet type with all displacement components set to zero).

First, we needed to determine an appropriate population size. We conducted a series of experiments with different population sizes $(10,20,50,100,500,1000)$. The same stopping criterion (0.001) as specified in Section IV-A was used. We started each simulation with a good initial guess to ensure that the solutions obtained are equally mechanically meaningful (close to the true solution). For each chosen population size, we ran 30 simulations and the average results are listed in Table $\mathrm{V}$ and plotted in Fig. 7.

The CGA with a larger population size can converge with many less generations than the CGA with a smaller population size but requires much more simulation time. In other words, for the CGA with a larger population size, each generation takes more time due to the computational cost associated with its large pool. Although the CGA with a smaller population size uses much less execution time, this benefit is often offset by the concern of premature convergence due to a less diversified pool that is common to smaller populations. Taking the above factors into account, we chose a population size of 100 which maintains enough population diversity yet still has a "reasonable" computational cost.

The behavior of a CGA that has $M$ chromosomes in its pool can be roughly viewed as being equivalent to $M$ local gradient methods that are run simultaneously. The difference is that the chromosomes in a CGA have certain degrees of information sharing among themselves through the crossover operation, while the gradient methods running in parallel are completely independent of each other. It would be interesting to compare
TABLE V

CGA EXPERIMENTS With DifFERENT POPUlation SizE. RESUlts ARE REPORTED AS THE AVERAGE of 30 Runs FOR EACH POPUlation SizE. All Simulations Were Started With a Good Initial Point

\begin{tabular}{|c||c|c|c|c|c|c|}
\hline Population Size & 10 & 20 & 50 & 100 & 500 & 1000 \\
\hline Generations & 2215 & 1032 & 764 & 422 & 339 & 128 \\
\hline Time (min) & 113 & 186 & 375 & 589 & 921 & 1556 \\
\hline
\end{tabular}

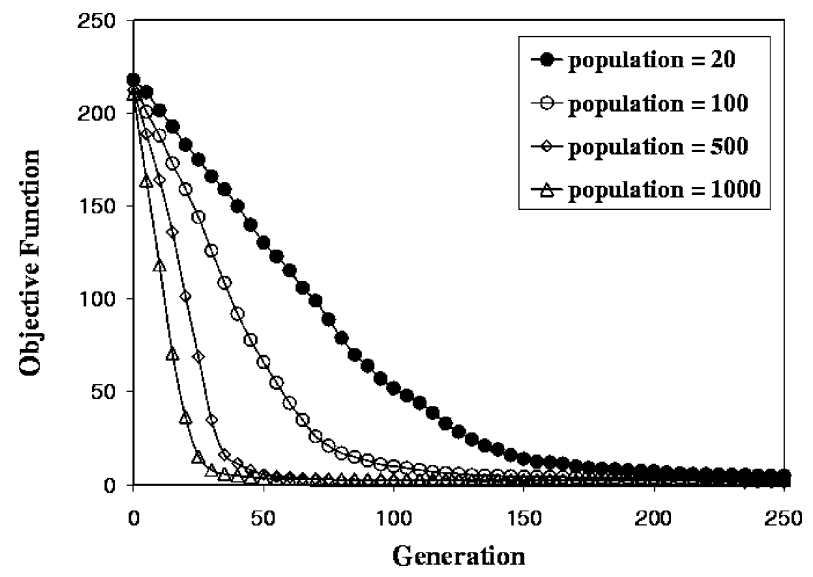

Fig. 7. The convergence behavior of the CGA with different population sizes. Each point on the curve is plotted using the average value of 30 simulations.

the performance of a CGA of $M$ chromosomes with that of $M$ independent gradient methods.

The gradient methods are known to be sensitive to the initial conditions. We use a guess that obeys the normal distribution law $\mathcal{N}(\mu, \sigma)$. In other words, the initial Young's modulus values assigned to 900 elements should have a sample mean of $\mu$ and a standard deviation of $\sigma$. We experimented with six such initial conditions (unit $=\mathrm{kPa}): \mathcal{N}(50,10), \mathcal{N}(100,20), \mathcal{N}(200,40)$, $\mathcal{N}(300,60), \mathcal{N}(400,80), \mathcal{N}(500,100)$, in the order of gradually deviating from the true solution. All of the experiments were performed on a Sun Sparc Ultra 5 machine (eight CPUs with $248 \mathrm{MHz}$ and $2560 \mathrm{Mb}$ ).

The experiments were designed as follows: for each of the six initial condition distributions, we ran 30 CGA simulations and 100 GNM simulations and present the results in Tables VI and VII. Each row in the tables presents the statistical performance of 30 CGA simulations or 100 GNM simulations, all with their initial guesses generated by the same normal distribution function. A CGA simulation was stopped if the convergence criterion as specified in (37) was below a threshold (0.001). A GNM simulation was stopped if the convergence criterion (38) was satisfied with a tolerance (1.0e-8) or a maximum iteration number was reached (400 in this experiment). We then evaluate the two algorithms based on five performance indexes: the convergence ratio, the number of evaluations of the objective function, the simulation time, and the averaged property values in the abnormal area and the background.

The convergence ratio is defined as the number of converged simulations divided by the total number of simulations. In the case of GNM, the drop in convergence ratio is noticeable as the initial condition moved away from the true solution. For example, starting with relatively poor initial conditions $(\mathcal{N}(400,50)$ and $\mathcal{N}(500,50))$, many GNM simulations failed to converge (or converged to a local minimum far away from 
TABLE VI

Performance of CGA ANd GNM With Different Initial Conditions. For EACH of the Six Initial Conditions, 30 CGa Simulations and 100 GNM Simulations Were Conducted. Results ARe REPORTED IN TERmS OF THE Number of EVAluations of ObJeCtive Function, the Simulation Time, and the Convergence Ratio. THE CONVERgence Ratio Is COMPuted as the Number of Converged Cases Divided by the Total Number of Simulations. OnLy Data of

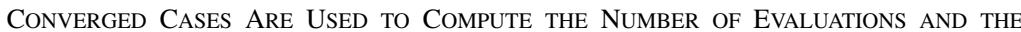
SiMULATION TIME. (SD $\rightarrow$ STANDARD DEVIATION)

\begin{tabular}{|c|c|c|c|c|c|c|c|c|c|c|c|}
\hline \multicolumn{12}{|c|}{ CGA } \\
\hline \multirow{2}{*}{$\begin{array}{l}\text { Init. Cond. } \\
\qquad \mathcal{N}(\mu, \sigma)\end{array}$} & \multicolumn{5}{|c|}{ Number of Evaluations } & \multicolumn{5}{|c|}{ Simulation Time (min) } & \multirow{2}{*}{$\begin{array}{c}\text { Converg } \\
\text { Ratio } \\
\end{array}$} \\
\hline & Min & Max & Mean & Median & SD & Min & $\operatorname{Max}$ & Mean & Median & SD & \\
\hline $\mathcal{N}(50,10)$ & 5100 & 8450 & 6524 & 6550 & 761 & 96 & 192 & 143 & 141 & 24 & $97 \%$ \\
\hline $\mathcal{N}(100,20)$ & 9500 & 12900 & 11378 & 11425 & 837 & 190 & 281 & 238 & 232 & 23 & $90 \%$ \\
\hline $\mathcal{N}(200,40)$ & 16350 & 19800 & 17713 & 17550 & 903 & 343 & 436 & 390 & 396 & 28 & $83 \%$ \\
\hline $\mathcal{N}(300,60)$ & 21150 & 25300 & 22954 & 23400 & 1255 & 457 & 526 & 501 & 502 & 26 & $76 \%$ \\
\hline $\mathcal{N}(400,80)$ & 24700 & 28950 & 26828 & 26725 & 1228 & 538 & 649 & 617 & 619 & 29 & $76 \%$ \\
\hline $\mathcal{N}(500,100)$ & 28100 & 40750 & 30736 & 30125 & 3137 & 602 & 747 & 632 & 624 & 37 & $73 \%$ \\
\hline \multicolumn{12}{|c|}{ GNM } \\
\hline Init. Cond. & \multicolumn{5}{|c|}{ Number of Evaluations } & \multicolumn{5}{|c|}{ Simulation Time (min) } & Converg \\
\hline $\mathcal{N}(\mu, \sigma)$ & Min & Max & Mean & Median & SD & Min & $\operatorname{Max}$ & Mean & Median & SD & Ratio \\
\hline $\mathcal{N}(50,10)$ & 1164 & 1507 & 1294 & 1204 & 136 & 27 & 40 & 34 & 32 & 4 & $98 \%$ \\
\hline $\mathcal{N}(100,20)$ & 1198 & 1628 & 1439 & 1518 & 152 & 31 & 48 & 41 & 39 & 5 & $82 \%$ \\
\hline $\mathcal{N}(200,40)$ & 1742 & 2054 & 1896 & 1932 & 173 & 46 & 76 & 70 & 68 & 7 & $63 \%$ \\
\hline $\mathcal{N}(300,60)$ & 2245 & 2791 & 2328 & 2296 & 168 & 44 & 93 & 86 & 82 & 6 & $44 \%$ \\
\hline $\mathcal{N}(400,80)$ & 2737 & 3652 & 2733 & 2705 & 202 & 59 & 108 & 91 & 93 & 7 & $32 \%$ \\
\hline $\mathcal{N}(500,100)$ & 2986 & 3893 & 3270 & 3361 & 218 & 74 & 122 & 95 & 103 & 8 & $28 \%$ \\
\hline
\end{tabular}

TABLE VII

Recovered Property Values With CGA and GNM. The Solutions Are Given in Terms of the Averaged Property Values in the Abnormal Area $\left(E_{a}\right)$ and the Background $\left(E_{b}\right)$. Statistical Data ARE BASED ON THE CONVERGED SimUlations ONLY

\begin{tabular}{|c|c|c|c|c|c|c|c|c|c|c|c|c|}
\hline \multicolumn{13}{|c|}{ CGA } \\
\hline \multirow{2}{*}{$\begin{array}{l}\text { Init. Cond. } \\
\mathcal{N}(\mu, \sigma)\end{array}$} & \multicolumn{6}{|c|}{ Abnormal area, $E_{a}(\mathrm{kPa})$} & \multicolumn{6}{|c|}{ Background, $E_{b}(\mathrm{kPa})$} \\
\hline & Min & Max & Mean & Median & SD & True & Min & $\operatorname{Max}$ & Mean & Median & SD & True \\
\hline $\mathcal{N}(50,10)$ & 189 & 412 & 259 & 264 & 41 & 400 & 50 & 56 & 53 & 53 & 2 & 50 \\
\hline $\mathcal{N}(100,20)$ & 193 & 573 & 366 & 337 & 129 & 400 & 52 & 58 & 54 & 54 & 3 & 50 \\
\hline $\mathcal{N}(200,40)$ & 192 & 598 & 397 & 376 & 162 & 400 & 54 & 59 & 56 & 56 & 2 & 50 \\
\hline$\overline{\mathcal{N}}(300,60)$ & 212 & 601 & 365 & 345 & 155 & 400 & 55 & 60 & 57 & 57 & 4 & 50 \\
\hline $\mathcal{N}(400,80)$ & 206 & 604 & 371 & 378 & 172 & 400 & 56 & 62 & 58 & 58 & 5 & 50 \\
\hline $\mathcal{N}(500,100)$ & 198 & 595 & 362 & 320 & 180 & 400 & 56 & 66 & 58 & 60 & 5 & 50 \\
\hline \multicolumn{13}{|c|}{ GNM } \\
\hline Init. Cond. & \multicolumn{6}{|c|}{ Abnormal area, $E_{a}(\mathrm{kPa})$} & \multicolumn{6}{|c|}{ Background, $E_{b}(\mathrm{kPa})$} \\
\hline $\mathcal{N}(\mu, \sigma)$ & Min & $\operatorname{Max}$ & Mean & Median & SD & True & Min & Max & Mean & Median & $\mathrm{SD}$ & True \\
\hline $\mathcal{N}(50,10)$ & 194 & 243 & 218 & 220 & 14 & 400 & 39 & 55 & 50 & 48 & 5 & 50 \\
\hline $\mathcal{N}(100,20)$ & 188 & 237 & 212 & 215 & 16 & 400 & 41 & 62 & 57 & 60 & 7 & 50 \\
\hline $\mathcal{N}(200,40)$ & 196 & 245 & 209 & 211 & 13 & 400 & 37 & 64 & 55 & 53 & 8 & 50 \\
\hline $\mathcal{N}(300,60)$ & 205 & 239 & 214 & 216 & 13 & 400 & 40 & 68 & 59 & 54 & 7 & 50 \\
\hline $\mathcal{N}(400,80)$ & 191 & 226 & 203 & 204 & 10 & 400 & 42 & 71 & 64 & 49 & 9 & 50 \\
\hline $\mathcal{N}(500,100)$ & 187 & 230 & 199 & 201 & 14 & 400 & 38 & 73 & 62 & 51 & 9 & 50 \\
\hline
\end{tabular}

the true solution). In contrast, the convergence ratio of the CGA is more stable. The performance deterioration of the CGA caused by a poor initialization is less drastic.

The gain in the convergence ratio of a CGA comes with a price of longer simulation time. A single CGA simulation took much more time to converge than a single GNM simulation did. This can also be seen in the number of evaluations, which are more or less proportional to the simulation time. On the other hand, if we view the outcome of a single CGA of 100 chromosomes as being equal to that of 100 independent GNMs, then running 100 independent GNMs seems more expensive than running a single CGA. However, this comparison could be misleading. It should be stressed that the bottleneck of a GNM simulation is the computation of the Jacobian matrix. In our current implementation, a finite difference approximation method is used, which implies that for a problem of $m$ parameters, at least $m^{2}$ calls of the forward model were needed for each iteration step. This accounts for about $87 \%$ of the total GNM simulation time. If a more efficient method such as the adjoint state method is used [46], the simulation time of a GNM can be significantly reduced, and the advantage of a single CGA against 100 independent GNMs will likely disappear. Furthermore, if we are dealing with a complex 3-D problem, a larger population size might be needed, which will also increase the computational cost of the CGA.

The averaged property values in the abnormal area $\left(E_{a}\right)$ and the background $\left(E_{b}\right)$ can provide valuable information about the quality of solutions (see Table VII). In the background, both CGA and GNM produced good results (small standard deviation and roughly the same mean and median values across different initial conditions). In the abnormal area, GNM solutions show more uniform distributions than CGA solutions. For ex- 


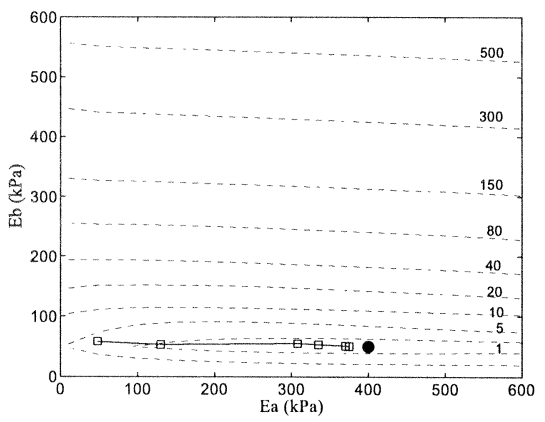

(a) GNM with initial guess: $E_{a}=48 \mathrm{kPa}, E_{b}=58 \mathrm{kPa}$

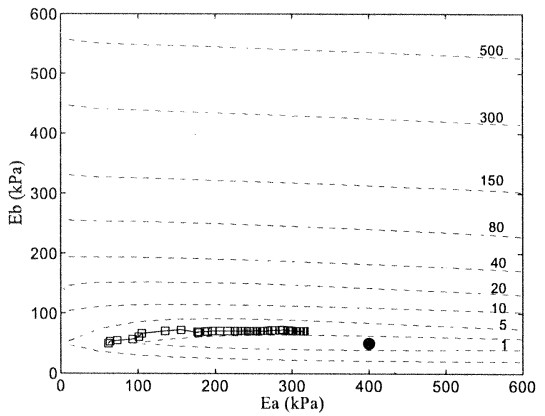

(c) CGA with initial guess: $E_{a}=62 \mathrm{kPa}, E_{b}=50 \mathrm{kPa}$

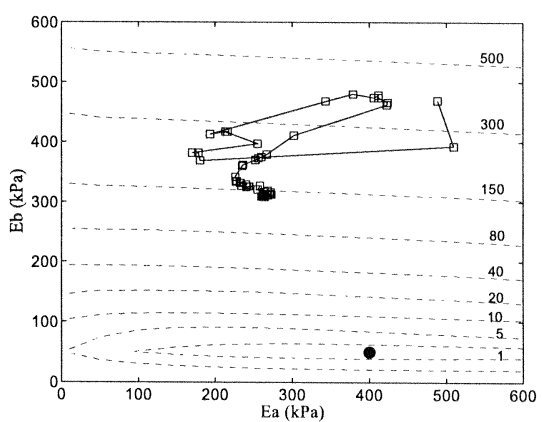

(b) GNM with initial guess:

$$
E_{a}=489 \mathrm{kPa}, E_{b}=469 \mathrm{kPa}
$$

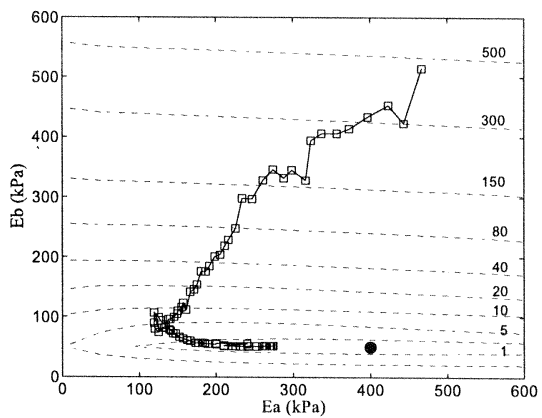

(d) CGA with initial guess: $E_{a}=467 \mathrm{kPa}, E_{b}=515 \mathrm{kPa}$

Fig. 8. Examples that show the convergence path of CGA and GNM simulations with different starting points. The contours (dashed lines) represent the values of objective function. The black circle indicates the position of the true solution. Note that this is a very simplified view of the solution space. The actual solution landscape in higher dimensions is much more complex and is characterized by numerous local minimums.

ample, GNM solutions are centered around $200 \mathrm{kPa}$ with small standard deviations, while CGA solutions vary over much large ranges (high standard deviations), even though the mean and median values of CGA are closer to the true solution (400 kPa). The is probably due to the fact that the smoothing effect of the quadratic constraint term in GNM is stronger than the piecewise and rank-based penalty function in CGA. It can also be seen that, given a good starting point $\mathcal{N}(50,10)$, solution statistics from the two algorithms become very similar.

From the above experimental results and analysis, several observations can be made.

1) The CGA is more robust in the sense that it is less sensitive to the initial conditions.

2) A single GNM is more efficient than a single CGA in terms of the convergence time. If a good initialization is available, the GNM is a more attractive choice.

3) A better solution strategy is to combine the GNM and the CGA in a way that both their strengths can be fully utilized. For instance, the CGA can be used to find a relatively good initial approximation, upon which the GNM can be launched to speed up the search.

4) The performance of an inverse algorithm can be affected by many factors, such as execution environment, gradient approximation method, implementation strategy, and the tolerance used to stop the simulation. Therefore, sound judgment based on a thorough understanding of the specific physical model is always needed.
To illustrate the convergence behaviors of the CGA and the GNM, we plot the minimization steps of four example runs in Fig. 8. Since it is difficult to visualize the solution landscape in high dimensions, we simplify the space in the following way: For each solution vector, we use the two averaged values $\left(E_{a}\right.$ and $E_{b}$ ) as new parameters to plot the solution and the corresponding objective function in a 2-D contour map. Each point in the 2-D map represents a possible solution. The true solution shown as a black circle is located at the global minimum of the solution space (objective function $=0$ ).

As shown in Fig. 8(a) and (c), if one started with a point close to the true solution, the GNM took only a few steps to reach the true solution, while the CGA could take a couple of hundred generations. Given a poor initial guess, Fig. 8(d) depicts the convergence path of a successful CGA simulation, while Fig. 8(b) shows a GNM simulation that failed to converge to the true solution (stuck in a local minimum).

It should be emphasized that Fig. 8 is only used for illustration. The actual solution space of 900 parameters is far more complex than the 2-D contour map. The are numerous local extrema that cannot be seen in this extremely simplified two parameter space.

We also plot the results of 100 GNM simulations in Fig. 9. The 100 examples are randomly picked from the total of 600 simulations (we had 100 simulations for each of the six initial conditions). The plot helps us visualize the influence of initialization on the behavior of local gradient methods. It is apparent 


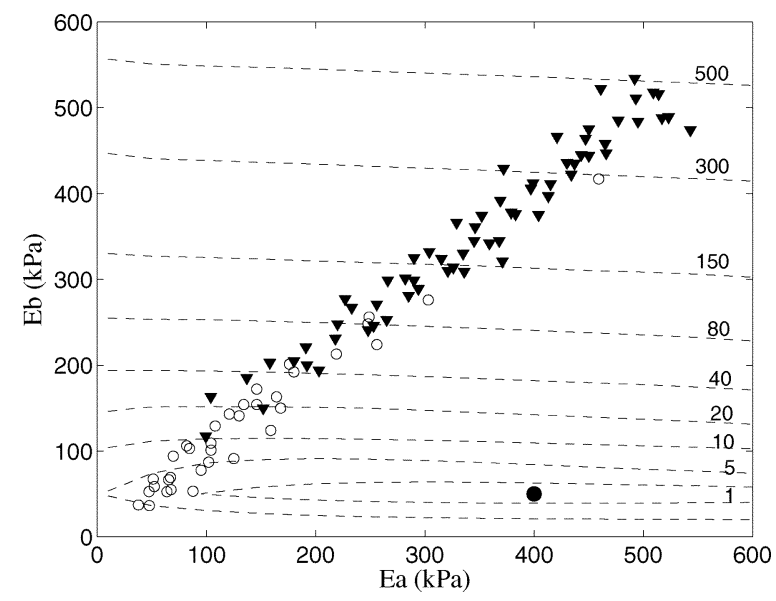

Fig. 9. The results of GNM simulations with various initial conditions. The circles and filled triangles represent the cases that converged and diverged, respectively.

that as the initial condition deviated away from the true solution, more and more GNM simulations failed to converge (trapped in local minimum).

\section{SUMMARY}

We present a constrained genetic approach for reconstructing the Young's modulus of elastic objects under deformation. This approach utilizes a rank-based mechanism to incorporate qualitative prior knowledge to constrain the admissible solutions. Experiments with noisy data show that the Young's modulus can be successfully reconstructed with significant improvement in both solution accuracy and stability. Based on the experiments with the GA, CGA, and GNM, several observations can be made about the following aspects of the recovery algorithms: 1) the robustness in the presence of data noise; 2) the computational efficiency; and 3) the sensitivity to initialization.

In all of the experiments, both the CGA and the GNM showed their robustness against data noise by converging to a stable and near-optimal solution, attributed to the constraint imposed through the penalty function. Being able to handle noisy data is critical to any reconstruction algorithm because ideal noise-free data is rarely available in practice.

Because of its stochastic nature, the CGA is more demanding than the gradient-based methods in terms of computational resources. For a 2-D nonlinear inverse problem of modest size, a population of more than 50 chromosomes and several hundreds of generations are necessary. For a more complex 3-D reconstruction problem, the demand for CPU power could be much higher.

Although the CGA is a computationally intensive method, it has a very desirable feature of being less sensitive to the initial conditions. By maintaining a diversified population pool, CGA can explore the solution space on a global scale without being caught in local extrema, which has been the main obstacle to the gradient-based methods. A recent study by Salomon [59] showed that a hybrid approach can yield better results than either a regular GA or a steepest descent method alone for certain test functions. The combination of genetic and Powell algorithms and its application in electrical impedance tomography was also reported [26]. A hybrid approach is certainly attractive for the Young's modulus reconstruction because the CGA can provide a good initial approximation by exploring a wide solution landscape, upon which a more efficient local gradient method can then be applied to expedite the convergence process. The study by Chiwiacowsky et al. [7] suggests that the hybrid method is very promising for solving the large-scale ill-posed elasticity reconstruction problem. But designing an appropriate framework to combine the two approaches and finding the optimal point at which the CGA is switched to the gradient method is still a challenging issue and future work is warranted. Another interesting approach is to embed the gradient method inside the evolutionary algorithm as a special operator. This method has the advantage that the switching between two algorithms and the associated overhead can be avoided [52]. Finally, it should be stressed that conclusions drawn from this study only apply to the particular setting of the inverse elastic problem with linear assumptions and should not be generalized to other domains without extensive tests using either real models or the well known benchmark functions.

\section{REFERENCES}

[1] S. Aglyamov, A. R. Skovoroda, J. M. Rubin, M. O’Donnell, and S. Y. Emelianov, "Model-based reconstructive elasticity imaging of deep venous thrombosis," IEEE Trans. Ultrason., Ferroelectr., Freq. Control, vol. 51, pp. 521-531, 2004.

[2] H. B. Ameur, M. Burger, and B. Hackl, "Level set methods for geometric inverse problems in linear elasticity," Inverse Prob., vol. 20, pp. 673-696, 2004.

[3] C. H. Arns, M. A. Knackstedt, W. V. Pinczewski, and E. J. Garboczi, "Computation of linear elastic properties from microtomographic images: Methodology and match to theory and experiment," J. Geophys., vol. 67, no. 5, pp. 1396-1405, 2002.

[4] P. E. Barbone and N. H. Gokhale, "Elastic modulus imaging: On the uniqueness and nonuniqueness of the elastography inverse problem in two dimensions," Inverse Prob., vol. 20, no. 1, pp. 283-296, 2004.

[5] C. C. A. Coello, "A survey of constraint handling techniques used with evolutionary algorithms," Laboratorio Nacional de Informatica Avanzada, Tech. Rep. Lania-RI-99-04, 1999.

[6] C. Chiroiu, L. Munteanu, V. Chiroiu, P. P. Delsanto, and M. Scalerandi, "A genetic algorithm for determination of the elastic constants of a monoclinic crystal," Inverse Prob., vol. 16, pp. 121-132, 2000.

[7] L. D. Chiwiacowsky, H. F. C. Velho, and P. Gasbarri, "A variational approach for solving an inverse vibration problem," in Inverse Problems, Design and Optimization Symp., Rio de Janeiro, Brazil, 2004.

[8] M. M. Doyley, P. M. Meaney, and J. C. Bamber, "Evaluation of an iterative reconstruction method for quantitative elastography," Phys. Med. Biol., vol. 45, pp. 1521-1540, 2000.

[9] R. Q. Erkamp, A. R. Skovoroda, S. Y. Emelianov, and M. O’Donnell, "Measuring the nonlinear elastic properties of tissue-like phantoms," IEEE Trans. Ultrason., Ferroelectr., Freq. Control, vol. 51, pp. 410-419, 2004.

[10] H. W. Engl, M. Hanke, and A. Neubauer, Regularization of Inverse Problems. Norwell, MA: Kluwer Academic, c1996.

[11] H. W. Engl, "Identification of parameters in polymer crystallization, semiconductor models and elasticity via iterative regularization methods," in Ill-Posed and Inverse Problems, V. G. Romanov, Ed. Leiden, The Netherlands: Brill, 2003.

[12] G. Eskin and J. Ralston, "On the inverse boundary value problem for linear isotropic elasticity," Inverse Prob., vol. 18, pp. 907-921, 2002.

[13] M. Fatemi, A. Manduca, and J. F. Greenleaf, "Imaging elastic properties of biological tissues by low-frequency harmonic vibration," Proc. IEEE, vol. 91, no. 10, pp. 1503-1519, 2003.

[14] M. Ferrant, A. Nabavi, B. Macq, E. A. Jolesz, R. Kikinis, and S. K. Warfield, "Registration of 3-D intraoperative MR images of the brain using a finite-element biomechanical model," IEEE Trans. Med. Imag., vol. 20, pp. 1384-1397, 2001.

[15] D. B. Fogel, Evolutionary Computation. Piscataway, NJ.: IEEE Press, 1995. 
[16] J. B. Fowlkes, S. Y. Yemelyanov, J. G. Pipe, P. L. Carson, R. S. Adler, A. P. Sarvazyan, and A. R. Skovoroda, "Possibility of cancer detection by means of measurement of elastic properties," Radiology, vol. 185, pp. 206-207, 1992.

[17] D. R. Franca and A. Blouin, "All-optical measurement of in-plane and out-of-plane Young's modulus and Poisson's ratio in silicon wafers by means of vibration modes," Meas. Sci. Technol., vol. 15, pp. 859-868, 2004.

[18] Y. C. Fung, Foundations of Solid Mechanics. Englewood Cliffs, NJ: Prentice-Hall, 1965.

[19] - Biomechanics; Mechanical Properties of Living Tissues, 2nd ed. Berlin, Germany: Springer-Verlag, 1993.

[20] T. Furukawa and G. Yagawa, "Inelastic constitutive parameters identification using an evolutionary algorithm with continuous individuals," Int. J. Numer. Meth. Eng., vol. 40, pp. 1071-1090, 1997.

[21] E. J. Garboczi and A. R. Day, "An algorithm for computing the effective linear elastic properties of heterogeneous materials: 3 -D results for composites with equal phase Poisson ratios," J. Phys. Mech. Solids, vol. 43, pp. 1349-1362, 1995.

[22] GMV (The General Mesh Viewer). Los Alamos National Laboratory. [Online]. Available: http://wwwxdiv.lanl.gov/XCM/gmv/GMVHome.html

[23] D. E. Goldberg, Genetic Algorithms in Search, Optimization and Machine Learning. Reading, MA.: Addison-Wesley, 1989.

[24] D. E. Goldberg, K. Deb, and B. Korb, "Do not worry, be messy," in Proc. 4th Int. Conf. Genetic Algorithms, San Mateo, CA, 1991, pp. 24-30.

[25] P. C. Hansen, "Analysis of discrete ill-posed problems by means of the L-curve," SIAM Rev., vol. 34, pp. 561-580, 1992.

[26] C. T. Hsiao, G. Chahine, and N. Gumerov, "Application of a hybrid genetic/Powell algorithm and a boundary element method to electrical impedance tomography," J. Comp. Phys., vol. 173, no. 2, pp. 433-454, 2001.

[27] M. Hori and K. Oguni, "Inverse analysis method for identification of local elastic properties by using displacement data," in Inverse Problems in Engineering Mechanics, M. Tanaka, Ed, Japan: Nagano, 2003, vol. IV, pp. 111-119.

[28] C.-H. Huang, "A nonlinear inverse vibration problem of estimating the time-dependent stiffness coefficients by conjugate gradient method," Int. J. Numer. Meth. Eng., vol. 50, no. 7, pp. 1545-1558, 2001.

[29] "Special issue on tissue motion and elasticity imaging," Phys. Med. Biol., vol. 45, no. 6, pp. 1409-1714, 2000.

[30] L. Ji and J. McLaughlin, "Recovery of the Lamé parameter in biological tissues," Inverse Prob., vol. 20, no. 1, pp. 1-24, 2004.

[31] A. John, W. Kus, and P. Orantek, "Material coefficients identification of bone tissues using evolutionary algorithms," in Inverse Problems in Engineering Mechanics, M. Tanaka, Ed, Japan: Nagano, 2003, vol. IV, pp. 95-101.

[32] A. Joukhadar, T. Garat, and C. Laugier, "Constraint-based identification of a dynamic model,' in Proc. Intelligent Robots Systems (IROS'97), vol. 1, 1997, pp. 337-342.

[33] F. Kallel and M. Bertrand, "Tissue elasticity reconstruction using linear perturbation method," IEEE Trans. Med. Imag., vol. 15, pp. 299-313, 1996.

[34] S. J. Kirkpatrick and D. D. Duncan, "Optical assessment of tissue mechanics," in Handbook of Optical Biomedical Diagnostics, V. Tuchin, Ed. Bellingham, WA: SPIE, 2002, submitted for publication.

[35] R. M. Koch, M. H. Gross, F. R. Carls, D. F. von Buren, G. Fankhauser, and Y. I. H. Parish, "Simulating facial surgery using finite element models," in Proc. SIGGRAPH 96, 1996, pp. 421-428.

[36] R. L. Maurice, J. Ohayon, Y. Fretigny, M. Bertrand, G. Soulez, and G. Cloutier, "Noninvasive vascular elastography: Theoretical framework," IEEE Trans. Med. Imag., vol. 23, pp. 164-180, 2004.

[37] E. Mezura-Montes and C. C. A. Coello, "An improved diversity mechanism for solving constrained optimization problems using a mutimembered evolution strategy," in Proc. Genetic Evolutionary Computation Conf. (GECCO'2004), vol. 3102, Lecture Notes in Computer Science, K. Deb et al., Eds., 2004, pp. 700-712.

[38] D. Metaxas, Physics-Based Deformable Models: Applications to Computer Vision, Graphics and Medical Imaging. Norwell, MA: Kluwer Academic, 1997.

[39] M. I. Miga, "A new approach to elastography using mutual information and finite elements," Phys. Med. Biol., vol. 48, pp. 467-480, 2003.

[40] K. Miller, "Least squares methods for ill-posed problems with prescribed bound," SIAM J. Math. Anal., vol. 1, pp. 52-74, 1970.

[41] Z. Michalewicz, "A survey of constraint handling techniques in evolutionary computation methods," in Proc. 4th Annu. Conf. Evolutionary Programming, J. R. McDonnell, R. G. Reynolds, and D. B. Fogel, Eds., 1995, pp. 135-155.
[42] V. A. Morozov, "On the solution of functional equations by the method of regularization,” Sov. Math. Dokl., vol. 7, pp. 414-417, 1966.

[43] R. Muthupillai, D. J. Lomas, P. J. Rossman, J. F. Greenleaf, A. Manduca, and R. L. Ehman, "Magnetic resonance elastography by direct visualization of propagating acoustic strain waves," Science, vol. 269, pp. 1854-1857, 1995.

[44] G. Nakamura and G. Uhlmann, "Identification of Lamé parameters by boundary measurements," Amer. J. Math., vol. 115, pp. 1161-1187, 1993.

[45] A. Neubauer and O. Scherzer, "Finite-dimensional approximation of Tikhonov regularized solutions of nonlinear ill-posed problems," Numer. Funct. Anal. Optim., vol. 11, pp. 85-99, 1990.

[46] A. A. Oberai, N. H. Gokhale1, and G. R. Feijoo, "Solution of inverse problems in elasticity imaging using the adjoint method," Inverse Prob., vol. 19, pp. 297-313, 2003.

[47] M. O'Donnell and A. R. Skovoroda, "Prospects for elasticity reconstruction in the heart," IEEE Trans. Ultrason., Ferroelectr., Freq. Control, vol. 51, no. 3, pp. 322-328, 2004.

[48] T. E. Oliphant, A. Manduca, R. L. Ehman, and J. F. Greenleaf, "Complex-valued stiffness reconstruction for magnetic resonance elastography by algebraic inversion of the differential equation," Magn. Res. Med., vol. 45, no. 2, pp. 299-310, 2001.

[49] R. Olmi, M. Bini, and S. Priori, "A genetic algorithm approach to image reconstruction in electrical impedance tomography," IEEE Trans. Evol. Comput., vol. 4, pp. 83-88, 2000.

[50] J. Ophir, I. Cespedes, H. Ponnekanti, Y. Yazdi, and X. Li, "Elastography: A quantitative method for measuring the elasticity of biological tissues," Ultrason. Imag., vol. 13, pp. 111-134, 1991.

[51] J. Ophir, S. K. Alam, B. S. Garra, F. Kallel, E. E. Konofagou, T. Krouskop, C. R. B. Merritt, R. Righetti, R. Souchon, S. Srinivasan, and T. Varghese, "Elastography: Imaging the elastic properties of soft tissues with ultrasound," J. Med. Ultrasound, vol. 29, pp. 155-171, 2002.

[52] P. Orantek, "Hybrid evolutionary algorithms in optimization of structures under dynamical loads," in Proc. IUTAM Symp. Evolutionary Methods Mechanics (SMIA), vol. 117, 2004.

[53] K. D. Paulsen, M. I. Miga, F. E. Kennedy, P. J. Hoopes, A. Hartov, and D. W. Roberts, "A computational model for tracking subsurface tissue deformation during sterotactic neurosurgery," IEEE Trans. Biomed. Eng., vol. 46, pp. 213-225, 1999.

[54] K. J. Parker, L. Gao, R. M. Lerner, and S. F. Levinson, "Techniques for elastic imaging: A review," IEEE Eng. Med. Biol., vol. 15, pp. 52-59, 1996.

[55] C. Pellot-Barakat, F. Frouin, M. F. Insana, and A. Herment, "Ultrasound elastography based on multiscale estimations of regularized displacement fields," IEEE Trans. Med. Imag., vol. 23, pp. 153-163, 2004.

[56] D. B. Plewes, J. Bishop, A. Samani, and J. Sciarretta, "Visualization and quantization of breast cancer biomechanical properties with magnetic resonance elastography," Phys. Med. Biol., vol. 45, no. 6, pp. 1591-1610, 2000.

[57] K. R. Raghavan and A. Yagle, "Forward and inverse problems in imaging the elasticity of soft tissue," IEEE Trans. Nucl. Sci., vol. 41, no. 4, pp. 1639-1647, 1994.

[58] T. P. Runarsson and X. Yao, "Stochastic ranking of constrained evolutionary optimization,” IEEE Trans. Evol. Comput., vol. 4, pp. 284-294, 2000.

[59] R. Salomon, "Evolutionary algorithms and gradient search: Similarities and differences," IEEE Trans. Evol. Comput., vol. 2, pp. 45-55, 1998.

[60] A. Samani, J. Bishop, and D. B. Plewes, "A constrained modulus reconstruction technique for breast cancer assessment," IEEE Trans. Med. Imag., vol. 20, pp. 877-885, 2001.

[61] C. G. Shaefer, "The ARGOT strategy: Adaptive representation genetic optimizer technique," in Proc. 2nd Int. Conf. Genetic Algorithms Applications, Hillsdale, NJ, 1987, pp. 50-55.

[62] C. Sumi and K. Nakayama, "A robust numerical solution to reconstruct a globally relative shear modulus distribution from strain measurements," IEEE Trans. Med. Imag., vol. 17, pp. 419-428, 1998.

[63] D. Terzopoulos and K. Waters, "Analysis and synthesis of facial image sequences using physical and anatomical models," IEEE Trans. Pattern Anal. Machine Intell., vol. 15, no. 6, pp. 569-579, 1993.

[64] L. V. Tsap, D. B. Goldgof, S. Sarkar, and P. S. Powers, "A vision-based technique for objective assessment of burn scars," IEEE Trans. Med. Imag., vol. 17, pp. 620-633, 1998.

[65] E. E. W. Van Houten, K. D. Paulsen, M. I. Miga, F. E. Kennedy, and J. B. Weaver, "An overlapping subzone technique for MR-based elastic property reconstruction," Magn. Res. Med., vol. 42, pp. 779-786, 1999. 
[66] S. Venkatraman and G. G. Yen, "A simple elitist genetic algorithm for constrained optimization," in Proc. Congr. Evolutionary Computation 2004 (CEC'2004), vol. 1, 2004, pp. 288-295.

[67] G. Wahba, "Spline models for observational data," in SIAM CBMS-NSF Regional Conf. Series Applied Mathematics, vol. 59, Philadelphia, PA, 1990.

[68] H. Wong and L. Guan, "Application of evolutionary programming to adaptive regularization in image restoration," IEEE Trans. Evol. Comput., vol. 4, no. 4, pp. 309-326, 2000.

[69] Y. Zhang, L. O. Hall, D. B. Goldgof, and S. Sarkar, "A constrained genetic approach for reconstructing Young's modulus of elastic objects from boundary displacement measurements," in Proc. 2002 Congr. Evolutionary Computation (CEC'02), vol. 1, 2002, pp. 1003-1008.

[70] Y. Zhu, T. J. Hall, and J. Jiang, "A finite-element approach for Young's modulus reconstruction," IEEE Trans. Med. Imag., vol. 22, pp. 890-901, 2003.

[71] O. C. Zienkiewicz, The Finite Element Method, 3rd ed. New York: McGraw-Hill, 1977.

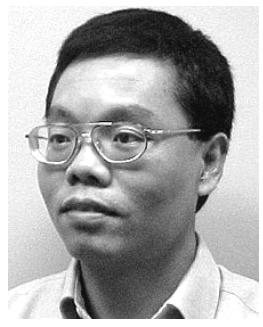

Yong Zhang received the $\mathrm{Ph} . \mathrm{D}$. degree in computer science and engineering from the University of South Florida, Tampa, in 2005.

$\mathrm{He}$ is currently an Assistant Professor in the Department of Computer Science and Information Systems, Youngstown State University, Youngstown, $\mathrm{OH}$. His research interests include computer vision, physics-based motion analysis, biomedical imaging, biometrics (face and gesture recognition), human-computer interaction, realistic animation, and evolutionary computations.

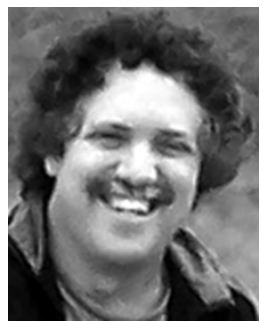

Lawrence O. Hall (M'84-F'03) received the B.S. degree in applied mathematics from the Florida Institute of Technology, Melbourne, in 1980 and the Ph.D. degree in computer science from Florida State University, Tallahassee, in 1986.

$\mathrm{He}$ is a Professor of Computer Science and Engineering at the University of South Florida, Tampa. He has authored over 190 publications in journals, conferences, and books. Recent publications appear in Artificial Intelligence in Medicine, Neural Computation, Pattern Recognition Letters, JAIR, Journal of Machine Learning Research, the IEEE TRANSACTIONS ON SYSTEMS, MAN, AND CYBERNETICS, the International Conference on Data Mining, the Multiple Classifier Systems Workshop, and the FUZZ-IEEE Conference (http://isl.csee.usf.edu/ailab/hall.html). He coedited the 2001 joint North American Fuzzy Information Processing Society (NAFIPS) and IFSA Conference Proceedings. He was the Co-Program Chair of NAFIPS 2004. $\mathrm{He}$ is a past President of NAFIPS. His research interests lie in distributed machine learning, data mining, pattern recognition and integrating AI into image processing. The exploitation of imprecision with the use of fuzzy logic in pattern recognition, AI, and learning is a research theme.

Prof. Hall received the IEEE SMC Society Outstanding Contribution Award in 2000. He received an Outstanding Research Achievement Award from the University of South Florida in 2004. He is a former Vice President for Membership of the IEEE SMC Society. He is the President of the SMC Society for 2006-2007. He was the Editor-in-Chief of the IEEE TRANSACTIONS ON Systems, MAN, AND CYBERNETICS-PART B, from 2002 to 2006. He is an Associate Editor of the IEEE TRANSACTIONS ON FUZZY SYSTEMS, the International Journal of Intelligent Data Analysis, and the International Journal of Approximate Reasoning.

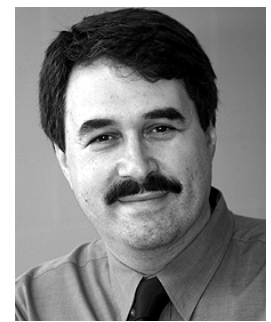

Dmitry Goldgof (M'83-SM'95) received the M.S. degree from Rensselaer Polytechnic Institute, Troy, $\mathrm{NY}$, in 1985 and the Ph.D. degree from the University of Illinois at Urbana-Champaign, Urbana, in 1989.

$\mathrm{He}$ is currently a Professor in the Department of Computer Science and Engineering and a member of $\mathrm{H}$. Lee Moffitt Cancer Center, where during 2002-2003, he was a Professor in Bioinformatics and Cancer Control. Previously, he held visiting positions at the University of California at Santa Barbara and the University of Bern, Switzerland He has graduated $12 \mathrm{Ph} . \mathrm{D}$. and 29 M.S. students, edited four books, and published 57 journal and over 100 conference papers. In 2002, he was granted a U.S. patent for his work in nonrigid motion analysis. His research interests include motion and deformation analysis, image analysis and its biomedical applications, bioinformatics, and pattern recognition.

Prof. Goldgof was awarded Annual Pattern Recognition Society Awards in 1993 and 2002. One of his papers was selected by the International Medical Informatics Association for its 2000 yearbook containing "The Best of Medical Informatics." He was named IEEE Distinguished Visitor in 2004. He is North American Editor for the Image and Vision Computing Journal and Associate Editor for the IEEE TRANSACTIONS ON SYSTEMS, MAN, AND CYBERNETICS. He has served as a member of the Editorial Board of Pattern Recognition, a member of the International Association of Pattern Recognition Education Committee, and Associate Editor for the IEEE TRANSACTIONS ON IMAGE PROCESSING.

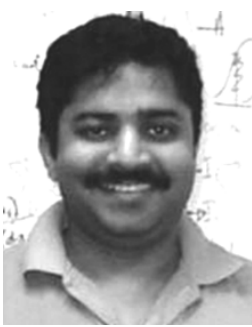

Sudeep Sarkar (M'93-SM'05) received the B.Tech. degree from the Indian Institute of Technology, Kanpur, in 1988, and the M.S. and Ph.D. degrees from The Ohio State University, Columbus, in 1990 and 1993, respectively, all in electrical engineering.

Since 1993, he has been with the Computer Science and Engineering Department, University of South Florida, Tampa, where he is currently a Professor. He has coauthored one book and coedited another book on perceptual organization. He was a Member of the Editorial Board of Pattern Analysis and Applications Journal (2000-2001) and is currently on the Editorial Board of Pattern Recognition. His research interests include perceptual organization in single images and multiple image sequences, biometrics, gait recognition, color-texture analysis, and performance evaluation of vision systems.

Prof. Sarkar received the National Science Foundation CAREER Award in 1994, the USF Teaching Incentive Program Award for undergraduate teaching excellence in 1997, the Outstanding Undergraduate Teaching Award in 1998 , and the Theodore and Venette Askounes-Ashford Distinguished Scholar Award in 2004. He received a University Presidential Fellowship from The Ohio State University. He was a Member of the Editorial Board of the IEEE TRANSACTIONS ON PATTERN ANALYSIS AND MACHINE INTELLIGENCE (1999-2003) and is currently on the Editorial Board of the IEEE TRANSACTIONS ON SYSTEMS, MAN, AND CYBERNETICS-PART B: CYBERNETICS. 